\title{
Global Analysis of Piecewise Linear Systems Using Impact Maps and Surface Lyapunov Functions
}

\author{
Jorge M. Gonçalves, Alexandre Megretski, and Munther A. Dahleh
}

\begin{abstract}
This paper presents an entirely new constructive global analysis methodology for a class of hybrid systems known as piecewise linear systems (PLS). This methodology infers global properties of PLS solely by studying the behavior at switching surfaces associated with PLS. The main idea is to analyze impact maps, i.e., maps from one switching surface to the next switching surface. Such maps are known to be "unfriendly" maps in the sense that they are highly nonlinear, multivalued, and not continuous. We found, however, that an impact map induced by an linear time-invariant flow between two switching surfaces can be represented as a linear transformation analytically parametrized by a scalar function of the state. This representation of impact maps allows the search for surface Lyapunov functions (SuLF) to be done by simply solving a semidefinite program, allowing global asymptotic stability, robustness, and performance of limit cycles and equilibrium points of PLS to be efficiently checked. This new analysis methodology has been applied to relay feedback, on/off and saturation systems, where it has shown to be very successful in globally analyzing a large number of examples. In fact, it is still an open problem whether there exists an example with a globally stable limit cycle or equilibrium point that cannot be successfully analyzed with this new methodology. Examples analyzed include systems of relative degree larger than one and of high dimension, for which no other analysis methodology could be applied. This success in globally analyzing certain classes of PLS has shown the power of this new methodology, and suggests its potential toward the analysis of larger and more complex PLS.
\end{abstract}

Index Terms-Hybrid systems, surface Lyapunov functions (SuLF), impact and Poincaré maps, global stability.

\section{INTRODUCTION}

YBRID systems are systems characterized by an interaction between continuous and discrete dynamics. The reason why we are interested in studying this class of systems is to capture discontinuity actions in the dynamics from either the controller or system nonlinearities. On one hand, a wide variety of physical systems are naturally modeled this way due to real-time changes in the plant dynamics like collisions, saturations, walking robots, cell cycle, etc. On the other hand, an engineer can introduce intentional nonlinearities to improve system performance, to effect economy in component selection, or to simplify the dynamic equations of the system by working with sets of simpler equations (e.g., linear) and switch among these

Manuscript received June 6, 2001; revised January 7, 2003, May 3, 2003, and June 13, 2003. Recommended by Associate Editor A. Bemporad.

J. M. Gonçalves is with the Control and Dynamical Systems Department, California Institute of Technology, Pasadena, CA 91125 USA (e-mail: jmg@cds.caltech.edu).

A. Megretski and M. A. Dahleh are with the Laboratory for Imformation and Decision Systems, the Massachusetts Institute of Technology, Cambridge, MA 92139 USA (e-mail: ameg@mit.edu; dahleh@mit.edu).

Digital Object Identifier 10.1109/TAC.2003.820061 simpler models (in order to avoid dealing directly with a set of nonlinear equations).

An important class of hybrid systems is piecewise linear systems (PLS). PLS are characterized by a finite number of linear dynamical models together with a set of rules for switching among these models. Therefore, this model description causes a partitioning of the state space into cells. These cells have distinctive properties in that the dynamics within each cell are described by linear dynamic equations. There are two main reasons why PLS are important. First, PLS are natural models for many applications like hopping robots, neural oscillators, and linear systems in feedback with static nonlinearities like relays and saturations. Second, PLS are much better approximations of nonlinear or hybrid systems than linear systems. A common practice in control is to study a linearization of a nonlinear system. Linear systems, however, only approximate the true dynamics in small regions around equilibrium points, and do not capture important properties of nonlinear systems like limit cycle oscillations.

Unlike linear systems, checking stability of general PLS is a very hard problem. For instance, it is not sufficient (nor necessary) that all linear subsystems are stable to guarantee stability of PLS [1]. Until very recently, very few results were available to analyze PLS. The work in [2] represents the first rigorous results to analyze general PLS. There, piecewise quadratic Lyapunov functions are constructed by solving a set of linear matrix inequalities (LMIs). This approach, however, can be inefficient or even unable to analyze many PLS, as discussed in detail in Section II, and motivates the need to develop new tools.

In [3], an entirely new methodology to globally analyze limit cycles of relay feedback systems (RFS) was introduced. The idea consisted of efficiently finding Lyapunov functions on switching surfaces to prove that Poincaré maps associated with the system were contracting. This paper generalizes these ideas to globally analyze PLS. The notion of Poincaré maps is generalized to impact maps, which are maps from one switching surface to the next switching surface, and not necessarily back to the original surface. The novelty of this work is in expressing impact maps induced by an LTI flow between two hyperplanes as linear transformations analytically parametrized by a scalar function of the state. Furthermore, level sets of this function are convex subsets of linear manifolds with dimension lower than that of the switching surfaces. This allows the search for surface Lyapunov functions (SuLF) to be done by solving sets of LMI's using efficient computational algorithms. Contractions of certain impact maps of the system can then be used to conclude about global stability, robustness, and performance of PLS. 
We will show that this new methodology can be used to not only globally analyze limit cycles but also equilibrium points of PLS. For that, on/off and saturation systems are analyzed, including those with unstable nonlinearity sectors for which classical methods like Popov criterion, Zames-Falb criterion [4], integral quadratic constraints (IQCs) [5]-[8], fail to analyze. In addition, the results in [9] and [10, Ch. 8] show that this methodology can also be efficiently applied to analyze robustness and performance of PLS. Thus, the success in globally analyzing stability, robustness, and performance of certain classes of PLS has shown the power of this new methodology, and suggests its potential toward the analysis of larger and more complex PLS.

This paper is organized as follows. The next section motivates the need for new analysis tools for PLS by explaining how available methods can be inefficient or even unable to analyze many PLS. Sections III and IV are dedicated to the development the main tool that relaxes the problem of checking contraction of impact maps to solving a semidefinite program. Then, Section V explains how this is used to globally analyze PLS. These results are applied to globally analyze asymptotic stability of on/off and saturation systems, in Sections VI and VII, respectively. Section VIII shows how less conservative global stability conditions can be obtained. Conclusions and future work are discussed in Section IX and, finally, technical details are considered in the Appendix.

\section{MOTIVATION}

As discussed in introduction, there exist several tools to analyze PLS. One of the most important consists of constructing piecewise quadratic Lyapunov functions (PQLFs) in the state-space [2], [11], [12]. This method relaxes the problem to a solution of a finite-dimensional set of LMIs. There are, however, several drawbacks with this approach that motivates the need for alternative methods to analyze PLS. These drawbacks are as follows.

- PQLF cannot analyze limit cycles since PQLF constructs Lyapunov functions in the state space.

- For most PLS, it is not possible to construct PQLF with just the given natural partition of the system. In order to improve flexibility, a refinement of partitions is typically necessary. The analysis method, however, is efficient only when the number of partitions required to prove stability is small. Example 2.1 below shows that even for second order systems, the construction of PQLF can be computationally intractable due to the large number of partitions in the state-space required for the analysis.

- In general, for systems of order higher than 3, it is extremely hard to obtain a refinement of partitions in the state-space to efficiently analyze PLS using PQLF. In other words, the method does not scale well with the dimension of the system. In fact, only a few and specific examples of PLS of order higher than 3 analyzed with this method have been reported.

- Existence of PQLF implies exponential stability of the system. Thus, PQLF cannot prove asymptotic stability of PLS that are not exponentially stable.

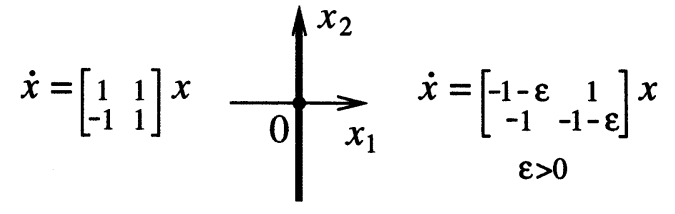

Fig. 1. PLS composed of an unstable and a stable linear systems.
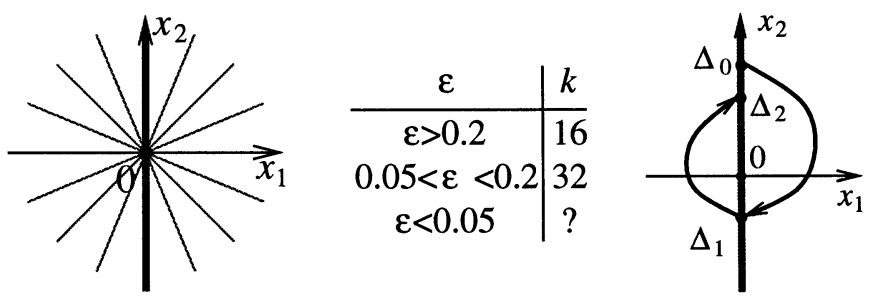

Fig. 2. State-space partitioned in 16 equal cells (left). Maps from one switch to the next switch (right).

Example 2.1: Consider the PLS in Fig. 1 composed of two linear subsystems. On the left side of the vertical $x_{2}$ axis there is an unstable linear system $\dot{x}=A_{u} x$ and on the right side a stable linear system $\dot{x}=A_{s} x$ parametrized by $\epsilon>0$. For this simple second order PLS, the goal is to show that the origin is globally asymptotically stable.

This PLS has no global quadratic Lyapunov function due to the unstable subsystem. We then turn to find PQLF. Starting with just the natural partition of the system, as expected no PQLF can be found, by the same reason there is no global quadratic Lyapunov function. A refinement of the state-space is then required. We decided to further partition the state space with $k$ equally separated lines through the origin, including the $x_{2}$ axis, resulting in $k$ equally sized partitions (see the left of Fig. 2 for $k=16$ ). Using the software developed by [2], for a given $\epsilon>0$ we tried successively $k=2,4,8,16,32, \ldots$ until the system could be successfully analyzed. The table in the center of Fig. 2 shows the smallest $k$ required to analyze the system as a function of $\epsilon$.

This table clearly shows that as $\epsilon$ decreases, the required number of partitions for the analysis of the PLS increases. For $\epsilon<0.05$, the number of required partitions is too high and it becomes computationally intractable to prove stability of the origin using this method. Note that even for large values of $\epsilon$, the smallest number of required partitions is already 16, although the original system was only divided in two partitions.

On the other hand, this system can easily analyzed on the switching surface without the need of extra complexity. In fact, it is easy to show that the maps from one switch to the next are contracting for any $\epsilon>0$ (see the right side of Fig. 2). Given $\epsilon, \Delta_{0}>0, \Delta_{1}=-e^{-\pi(1+\epsilon)} \Delta_{0}$ and $\Delta_{2}=-e^{\pi} \Delta_{1}$. Thus, $\Delta_{2}=e^{-\pi \epsilon} \Delta_{0}$. Since $e^{-\pi \epsilon}<1$ for all $\epsilon>0$, the origin is globally asymptotically stable for all $\epsilon>0$.

As we will see later, all the drawbacks of PQLF discussed above are not an issue for the classes of PLS analyzed so far using SuLF. First, SuLF can analyze both limit cycles [3] and equilibrium points (Sections VI and VII). Second, it is sufficient to consider only the natural partition of the system, with no extra complexity added. Third, our new method scales with 
the dimension of the system, and, finally, SuLF can be used to prove global asymptotic stability of PLS that are not exponentially stable (see example 7.3).

Also, the construction of PQLF for PLS proposed in [2] imposes continuity of the the Lyapunov functions along switching surfaces. This means that the intersection of two quadratic Lyapunov functions with a switching surface-one from each side-defines a unique quadratic Lyapunov function on the switching surface. Therefore, existence of PQLF guarantee the existence of SuLF. The converse, however, is not true. For instance, SuLF exist to analyze limit cycles [3], but no PQLF exist in the state-space.

Analysis of PLS at switching surfaces requires the understanding of system trajectories. When a trajectory leaves a switching surface it will either not switch again or switch in finite time (see Fig. 3). If the trajectory does not switch again then its behavior from thereon is simply governed by a linear system. Thus, linear analysis tools can be applied to this trajectory to check whether or not this will converge to an equilibrium point.

Things become more interesting when a trajectory leaving a switching surface does switch in finite time. This raises several questions: what happens to the trajectory after it switches? Will it switch again? Will it converge to some equilibrium point or limit cycle? These are the type of questions we address in this paper. To answer them, we must first fully understand a single map from one switching surface to the next switching surface (Sections III and IV). Then, PLS can be analyzed by carefully combining the analysis of all switching maps associated with the system (Section V).

\section{IMPACT MAPS}

Analysis of oscillations in nonlinear or hybrid systems systems is typically done with the so-called Poincaré map. A Poincaré map reduces the study of an $n$-dimensional system to a discrete $n-1$-dimensional system in a manifold (see, for example, [13]). The problem with Poincaré maps is that, in general, they cannot be found explicitly and are typically nonlinear, multivalued, and not continuous. Thus, global analysis of PLS is rarely done using these maps. Next, we explain how these difficulties inherent to Poincaré maps can be overcome to globally analyze PLS.

First, we need to understand the behavior of the system as this flows from one switching surface to the next switching surface. A useful notion that will be used throughout this paper is that of impact map, which is a generalization of a Poincaré map. An impact map is a map from one switching surface to the next switching surface, and not necessarily back to the original switching surface as in Poincaré maps. Only after we understand how to efficiently analyze a single impact map can we look at PLS as a whole, by combining all impact maps associated with the PLS, to conclude about stability, robustness, and performance properties of the system.

Consider the following affine linear time-invariant system

$$
\dot{x}=A x+B
$$

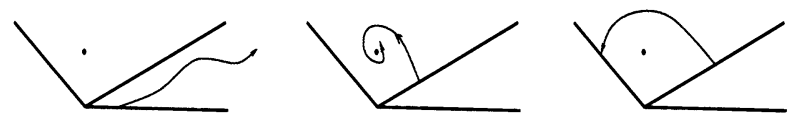

Fig. 3. Possible scenarios for a trajectory entering a cell: not convergent or unstable, stable, and switching trajectory.

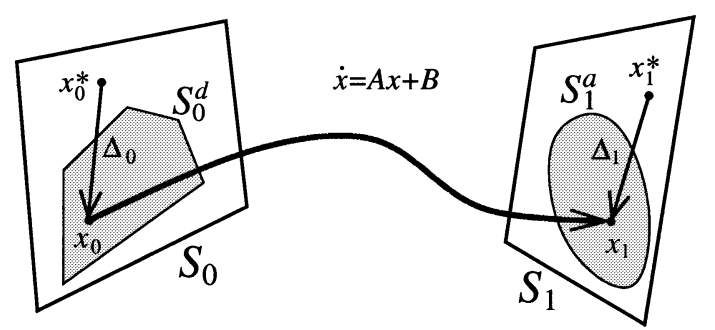

Fig. 4. Impact map from $\Delta_{0} \in S_{0}^{d}-x_{0}^{*}$ to $\Delta_{1} \in S_{1}^{a}-x_{1}^{*}$.

where $x \in \mathbb{R}^{n}, A \in \mathbb{R}^{n \times n}$, and $B \in \mathbb{R}^{n}$. Note that there are no restrictions on $A$. This matrix is allowed to have stable, unstable, and pure imaginary eigenvalues. Assume (1) is part of some larger PLS, and that (1) is defined on some open polytopical set $X \subset \mathbb{R}^{n}$. Consider the following hyperplanes in the boundary of $X$ :

$$
S_{0}=\left\{x \in \mathbb{R}^{n}: C_{0} S x=d_{0}\right\} \quad S_{1}=\left\{x \in \mathbb{R}^{n}: C_{1} x=d_{1}\right\} .
$$

Assume a trajectory arrived at $t=0$ in a subset of $S_{0}$ and the PLS switches to system (1). The impact map of interest is a map from some subset of $S_{0}$ to some subset of $S_{1}$.

More rigorously, let $\bar{X}$ denote the closure of $X$. Let $S_{0}^{d}$ be some polytopical subset of $S_{0}$ where any trajectory starting at $S_{0}^{d}$ satisfies $x(t) \in S_{1}$, for some finite $t \geq 0$, and $x(\tau) \in \bar{X}$ on $[0, t]$. Let also $S_{1}^{a} \subset S_{1}$ be the set of those points $x_{1}=x(t)$. The set $S_{1}^{a}$ can be seen as the image set of $S_{0}^{d}$ (see Fig. 4).

We are interested in studying the impact map, induced by (1), from $x_{0} \in S_{0}^{d}$ to $x_{1} \in S_{1}^{a}$. Since both $x_{0}$ and $x_{1}$ belong to switching surfaces, they can be parametrized in their respective hyperplanes. For that, let $x_{0}=x_{0}^{*}+\Delta_{0}$ and $x_{1}=x_{1}^{*}+\Delta_{1}$, where $x_{0}^{*} \in S_{0}, x_{1}^{*} \in S_{1}$, and $\Delta_{0}, \Delta_{1}$ are any vectors such that $\Delta_{0} \in S_{0}^{d}-x_{0}^{*}$ and $\Delta_{1} \in S_{1}^{a}-x_{1}^{*}$. In this case, $C_{0} \Delta_{0}=$ $C_{1} \Delta_{1}=0$. Note that $x_{0}^{*}$ and $x_{1}^{*}$ do not need to belong to $S_{0}^{d}$ and $S_{1}^{a}$, respectively. In fact, as explained later, in many cases is convenient to choose $x_{0}^{*} \in S_{0}$ so that $x_{0}^{*} \notin S_{0}^{d}$. Define also $x_{0}^{*}(t)$ as the trajectory of (1), starting at $x_{0}^{*}$, for all $t \geq 0$. The impact map of interest reduces to the map from $\Delta_{0}$ to $\Delta_{1}$ (see Fig. 4)

Note that, in general, the impact map from $\Delta_{0} \in S_{0}^{d}-x_{0}^{*}$ to $\Delta_{1} \in S_{1}^{a}-x_{1}^{*}$ defined above is multivalued and not continuous. This is illustrated in the following example.

Example 3.1: Consider a third-order system given by

$$
\dot{x}=\left(\begin{array}{ccc}
-1 & 0 & 0 \\
0 & -2 & 0 \\
0 & 0 & -3
\end{array}\right) x+\left(\begin{array}{l}
1 \\
1 \\
1
\end{array}\right)
$$

with the switching surfaces defined previously given by $C_{0}=$ $C_{1}=\left[\begin{array}{lll}-2 & 2 & 1\end{array}\right]$, and $d_{0}=0.5, d_{1}=-0.5$. Let $X=\left\{x \mid d_{1}<\right.$ $\left.C_{1} x(t)<d_{0}\right\}$. In the state space, the switching surfaces are parallel to each other. Let $x(0)=\left[\begin{array}{lll}-0.7-4.35 & 7.8\end{array}\right]^{\prime} \in S_{0}$. The resulting $C_{1} x(t)$ can be seen on the left of Fig. 5. 

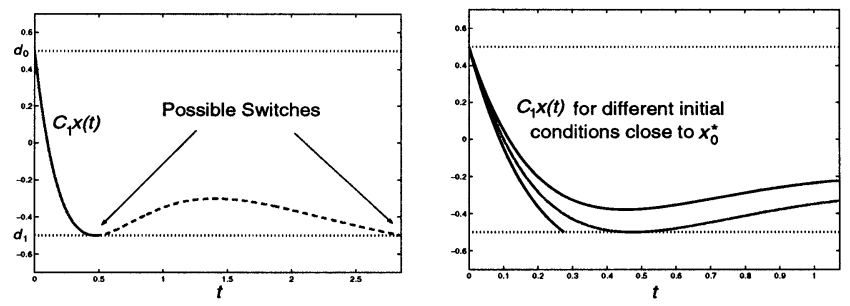

Fig. 5. Existence of multiple solutions (left). Map from $\Delta_{0}$ to $\Delta_{1}$ is not continuous (right).

When $t \approx 0.47, C_{1} x(t)=d_{1}$ and $C_{1} \dot{x}(t-0)=0$. At this point, the trajectory can return to $X$ (dashed trajectory), or it can switch. This means that a switch can occur at either $t=0.47$ or $t=2.85$, showing that the impact map is multivalued.

Now, let $x_{0}^{*}=x(0)$ and $x_{1}^{*}=x(0.47)$. The impact map from $\Delta_{0}$ to $\Delta_{1}$, as defined previously, is also not continuous since there is no neighborhood of $x_{0}^{*}$ that is mapped to a small enough neighborhood of $x_{1}^{*}$ (see the right-hand side of Fig. 5).

Definition 1: Let $x(0)=x_{0}^{*}+\Delta_{0} \in S_{0}^{d}$. Define $t_{\Delta_{0}}$ as the set of all times $t_{i} \geq 0$ such that the trajectory $x(t)$ with initial condition $x(0)$ satisfies $x\left(t_{i}\right) \in S_{1}$ and $x(t) \in \bar{X}$ on $\left[0, t_{i}\right]$. Define also the set of switching times of the impact map from $\Delta_{0} \in S_{0}^{d}-x_{0}^{*}$ to $\Delta_{1} \in S_{1}^{a}-x_{1}^{*}$ as

$$
\mathcal{T}=\left\{t \mid t \in t_{\Delta_{0}}, \quad \Delta_{0} \in S_{0}^{d}-x_{0}^{*}\right\}
$$

For instance, in Example 3.1, $t_{\Delta_{0}}=\{0.47,2.85\}$ for $x(0)$.

Besides from being multivalued and not continuous, impact maps are also typically nonlinear. Given an initial condition $\Delta_{0} \in S_{0}^{d}-x_{0}^{*}$, finding a $\Delta_{1} \in S_{1}^{a}-x_{1}^{*}$ requires first to find an associated switching time $t \in t_{\Delta_{0}}$. Solving for such $t$, however, involves solving a transcendental equation, which cannot, in general, be written in a closed form. Thus, numerical procedures are typically the only way to solve for $t$. This "nonfriendly" nature of impact maps and Poincaré maps in general is the main reason why global analysis of such maps has not been done before. The following result, however, shows that impact maps have a special structure that opens the door to analysis of PLS at switching surfaces.

Theorem 1: Assume $C_{1} x_{0}^{*}(t) \neq d_{1}$ for all $t \in \mathcal{T}$. Define

$$
\begin{aligned}
w(t) & =\frac{C_{1} e^{A t}}{d_{1}-C_{1} x_{0}^{*}(t)} \text { and } \\
H(t) & =e^{A t}+\left(x_{0}^{*}(t)-x_{1}^{*}\right) w(t)
\end{aligned}
$$

Then, for any $\Delta_{0} \in S_{0}^{d}-x_{0}^{*}$ there exists a $t \in \mathcal{T}$ such that the impact map is given by

$$
\Delta_{1}=H(t) \Delta_{0}
$$

Such $t \in t_{\Delta_{0}}$ is the switching time associated with $\Delta_{1}$.

This theorem says that maps between switching surfaces, induced by LTI flows, can be represented as linear transformations analytically parametrized by the correspondent switching time. At first, (2) may not seem of great help in analyzing the impact map. $\Delta_{1}$ is a linear function of $\Delta_{0}$ and a nonlinear function of the switching time $t$. The switching time, however, is a nonlinear function of $\Delta_{0}$ and a transcendental equation still needs to be solved in order to find $t$. Thus, by this reasoning, it seems (2) does not bring anything new.

This is, however, just one way of thinking about (2). Fortunately, there is another way to approach this equation. Assume, for now, the switching time $t$ is fixed. The result: impact map (2) is linear. Hence, the question is: what does it mean to have the switching time $t$ fixed? In other words, what are the set of points $x_{0}^{*}+\Delta_{0}$ in $S_{0}^{d}$ with the same switching time of $t$ ? In that set, impact map (2) is linear.

It turns out that the set of points in $S_{0}^{d}$ that has the same switching time $t$ is a convex subset of a linear manifold of dimension $n-2$ (see Fig. 6). Let $S_{t}$ be that set, that is, the set of points $x_{0}^{*}+\Delta_{0} \in S_{0}^{d}$ such that $t \in t_{\Delta_{0}}$. In other words, a trajectory starting at $x_{0} \in S_{t}$ satisfies both $x(t) \in S_{1}$ and $x(\tau) \in \bar{X}$ on $[0, t]$. Note that since the impact map is multivalued, a point in $S_{0}^{d}$ may belong to more than one set $S_{t}$. In fact, in Example 3.1, there existed a point in $S_{0}^{d}$ that belonged to both $S_{0.47}$ and $S_{2.85}$.

Now, as $t \in \mathcal{T}$ changes, $S_{t}$ covers every single point of $S_{0}^{d}$, i.e., $S_{0}^{d}=\left\{x \mid x \in S_{t}, t \in \mathcal{T}\right\}$. This follows since every point $\Delta_{0} \in S_{0}^{d}-x_{0}^{*}$ can switch for the first time at $S_{1}^{a}$, and therefore $t_{\Delta_{0}}$ is always a nonempty set. In other words, $S_{t}$ is an equivalence class of all initial conditions in the domain of the impact map with the same switching time. These results are summarized in the following corollary, which, as explained in the next section, is fundamental to relax the problem of stability, robustness, and performance analysis of PLS using SuLF to the solution of a semidefinite program.

Corollary 1: Under the assumptions of Theorem 1, for a given $t \in \mathcal{T}$, the impact map from $\Delta_{0} \in S_{t}-x_{0}^{*}$ to $\Delta_{1} \in$ $S_{1}-x_{1}^{*}$, given by $\Delta_{1}=H(t) \Delta_{0}$, is linear. Moreover, $S_{t}$ is a subset of a linear manifold of dimension $n-2$, and $S_{0}^{d}=$ $\left\{x \mid x \in S_{t}, t \in \mathcal{T}\right\}$.

Before proving the above results, it is important to understand the meaning of the assumption in Theorem 1 . This says the trajectory $x_{0}^{*}(t)$ cannot intersect the switching surface $S_{1}$ for all $t \in \mathcal{T}$. Note that no assumptions have been made on $x_{0}^{*}$ (the initial condition of $x_{0}^{*}(t)$ ), except that $x_{0}^{*} \in S_{0}$. In many cases, a careful choice of $x_{0}^{*} \in S_{0}$ is enough to satisfy the assumption (see Sections VI and VII). In some cases, however, either $x_{0}^{*}$ cannot be freely chosen (like in [3]) or there is simply no choice of $x_{0}^{*} \in S_{0}$ that satisfies the assumption. This means there exist at least one $t_{s} \in \mathcal{T}$ such that $C_{1} x_{0}^{*}\left(t_{s}\right)=d_{1}$. In such cases, the results in this paper still hold but with a slightly more complicated proof. For some PLS, like in [3], $w(t)$ at $t=t_{s}$ is defined as the limit when $t \rightarrow t_{s}$ (see [3] for details). If this is not the case, at $t=t_{s}$ the impact map can still be written as a linear transformation but parametrized by an extra variable, i.e., $\Delta_{1}=H_{s}\left(t_{s}, \delta\right) \Delta_{0}$, with $\Delta_{0} \in S_{t_{s}}$.

Proof of Theorem 1: Let $x(0)=x_{0} \in S_{0}^{d}$. Integrating the differential equation (1) gives

$$
x_{1}=e^{A t} x_{0}+\int_{0}^{t} e^{A(t-\tau)} B d \tau .
$$

Since $x_{0}=x_{0}^{*}+\Delta_{0}$ and $x_{1}=x_{1}^{*}+\Delta_{1}$,

$$
\begin{aligned}
\Delta_{1} & =e^{A t} \Delta_{0}+e^{A t} x_{0}^{*}+\int_{0}^{t} e^{A(t-\tau)} B d \tau-x_{1}^{*} \\
& =e^{A t} \Delta_{0}+x_{0}^{*}(t)-x_{1}^{*} .
\end{aligned}
$$




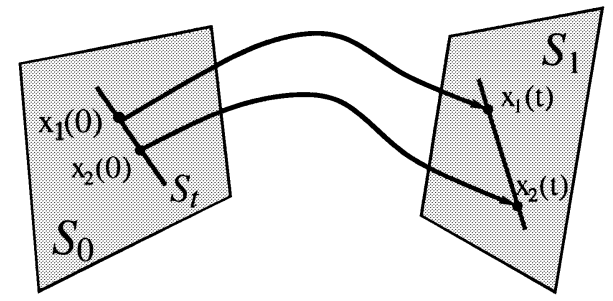

Fig. 6. Every point in $S_{t}$ has a switching time of $t$.

From the fact $C_{1} \Delta_{1}=0$ and $C_{1} x_{1}^{*}=d_{1}$

$$
C_{1} e^{A t} \Delta_{0}=d_{1}-C_{1} x_{0}^{*}(t) .
$$

Since, by assumption, $C_{1} x_{0}^{*}(t) \neq d_{1}$ for all $t \in \mathcal{T}$, the last expression can be written as

$$
w(t) \Delta_{0}=1
$$

which means $\Delta_{1}$ reduces to

$$
\Delta_{1}=e^{A t} \Delta_{0}+\left(x_{0}^{*}(t)-x_{1}^{*}\right) w(t) \Delta_{0}
$$

which proves the desired result.

Note that if $A$ is invertible, $x_{0}^{*}(t)$ can be written as $x_{0}^{*}(t)=$ $e^{A t}\left(x_{0}^{*}+A^{-1} B\right)-A^{-1} B$.

Proof of Corollary 1: The only thing left to prove is that $S_{t}$ is a subset of a linear manifold of dimension $n-2$. Let $x_{0}=x_{0}^{*}+\Delta_{0} \in S_{t}$. Since $C_{1} x(t)=d_{1}, \Delta_{0}$ must satisfy (3). Also, $C_{0} \Delta_{0}=0$ since $\Delta_{0} \in S_{0}-x_{0}^{*}$. Thus, since both equalities are linear on $\Delta_{0}, S_{t}-x_{0}^{*}$ has at most dimension $n-2$ and is a subset of a linear manifold .

\section{SURFACE LyAPUNOV FUNCTIONS}

As explained before, there are some results to construct piecewise quadratic Lyapunov functions for PLS [2]. Although these results are able to analyze equilibrium points of certain classes of PLS, many important PLS cannot be analyzed this way because they either have limit cycles or the method is computationally too expensive.

An alternative to construct Lyapunov functions in the state space is to construct Lyapunov functions on switching surfaces (SuLF). Define then two Lyapunov functions $V_{0}$ and $V_{1}$ on the switching surfaces $S_{0}^{d} \subset S_{0}$ and $S_{1}^{a} \subset S_{1}$, respectively. If

$$
V_{1}\left(\Delta_{1}\right)<V_{0}\left(\Delta_{0}\right) \text { for all } \Delta_{0} \in S_{0}^{d}-x_{0}^{*}
$$

then the impact map from $S_{0}^{d}$ to $S_{1}^{a}$ is a contraction. Checking (5) is, in general, very hard due to the nonlinear nature of impact maps. However, by using the results from the previous section and choosing the Lyapunov function candidates $V_{i}$ to be polynomial, contraction of impact maps can be relaxed to a matrix inequality. Let $P>0$ on $S$ stand for $x^{\prime} P x>0$ for all nonzero $x \in S$.

Theorem 2: Let $V_{i}$ be polynomial Lyapunov functions. There exists a matrix $R(t)$ parametrized by the switching time $t \in \mathcal{T}$ such that the impact map from $\Delta_{0} \in S_{0}^{d}-x_{0}^{*}$ to $\Delta_{1} \in S_{1}^{a}-x_{1}^{*}$ is a contraction if

$$
R(t)>0 \text { on } S_{t}-x_{0}^{*}
$$

for all switching times $t \in \mathcal{T}$.
The idea of the proof is simple. Substitute (2) in (5), use the fact that the impact map is linear in $S_{t}$, and that, as $t$ ranges over $\mathcal{T}, S_{t}$ covers every point in $S_{0}^{d}$.

If the SuLF $V_{0}$ and $V_{1}$ are chosen quadratic of the form

$$
V_{i}(x)=x^{\prime} P_{i} x-2 x^{\prime} g_{i}+\alpha_{i}
$$

for $i=0,1$, where $P_{i}>0, g_{i}$, and $\alpha_{i}$ are parameters to be found, then Theorem 2 reduces to the next corollary. As a short hand, denote $H_{t}$ for $H(t)$ and $w_{t}$ for $w(t)$.

Corollary 2: Let $V_{i}$ be quadratic Lyapunov functions as in (7) and define

$$
R(t)=P_{0}-H_{t}^{\prime} P_{1} H_{t}-2\left(g_{0}-H_{t}^{\prime} g_{1}\right) w_{t}+w_{t}^{\prime} \alpha w_{t}
$$

where $\alpha=\alpha_{0}-\alpha_{1}$. The impact map from $\Delta_{0} \in S_{0}^{d}-x_{0}^{*}$ to $\Delta_{1} \in S_{1}^{a}-x_{1}^{*}$ is quadratically stable if and only if there exist $P_{0}, P_{1}>0$ and $g_{0}, g_{1}, \alpha$ such that $R(t)>0$ on $S_{t}-x_{0}^{*}$ for all switching times $t \in \mathcal{T}$.

Condition (6) is not yet an LMI, which can be efficiently solved using available software. There are several ways to relax (6) to an semidefinite program. A more conservative condition than (6) results when the set of initial conditions $S_{t}$ is relaxed to

$$
R(t)>0 \text { on } S_{0}^{d}-x_{0}^{*}
$$

If this condition is satisfied then (6) follows since $S_{t} \subset S_{0}^{d}$. A trivial way to obtain a set of LMIs is to further relax the set of initial conditions. Although it results in a more conservative condition, this is also computationally more efficient.

Corollary 3: The impact map from $\Delta_{0} \in S_{0}^{d}-x_{0}^{*}$ to $\Delta_{1} \in$ $S_{1}^{a}-x_{1}^{*}$ is a contraction if

$$
R(t)>0 \text { on } S_{0}-x_{0}^{*}
$$

for all switching times $t \in \mathcal{T}$.

We have then relaxed the problem of contraction of impact maps to the solution of an infinite dimensional set of LMIs. As shown in several examples in Sections VI and VII, and also in [3], although condition (8) is more conservative than (6), in many situations it is enough to efficiently and successfully globally analyze PLS. Section VIII explains how to relax condition (6) to less conservative sets of LMIs.

For all $t \in \mathcal{T}$, condition (8) is an infinite set of LMIs. Computationally, this set is gridded to obtain a finite subset of LMIs, consisting of (8) on the switching times $t=\left\{t_{i}\right\} \subset \mathcal{T}$. For some large enough set $\left\{t_{i}\right\}$, it can be shown that (8) is also satisfied for all $t \in \mathcal{T}$. The idea is to find bounds on the derivative of the minimum eigenvalue of $R(t)$ over $\left(t_{i}, t_{i+1}\right)$, and then use these bounds to show nothing can go wrong in the intervals $\left(t_{i}, t_{i+1}\right)$, i.e., that (8) is also satisfied on each interval $\left(t_{i}, t_{i+1}\right)$ (see [3] for more details).

Note that, for a given $t$, condition (8) reduces to an $n-$ 1-dimensional LMI. Thus, an increase in the dimension of the system only results in proportionally larger LMIs. Hence, the stability condition (8) scales with the dimension of the system.

Next is the proof of corollary 2 (quadratic SuLF). The proof of Theorem 2 (polynomial SuLF) follows similarly using the results from [14] to efficiently express positivity of polynomials as sum of squares. 

orem 1

Proof of Corollary 2: From (5) and (7), and using The-

$$
\begin{aligned}
& \Delta_{1}^{\prime} P_{1} \Delta_{1}-2 \Delta_{1}^{\prime} g_{1}+\alpha_{1}<\Delta_{0}^{\prime} P_{0} \Delta_{0}-2 \Delta_{0}^{\prime} g_{0}+\alpha_{0} \\
& \quad \Leftrightarrow \Delta_{0}^{\prime}\left(P_{0}-H_{t}^{\prime} P_{1} H_{t}\right) \Delta_{0}-2 \Delta_{0}^{\prime}\left(g_{0}-H_{t}^{\prime} g_{1}\right)+\alpha>0 .
\end{aligned}
$$

Finally, using (4), we have

$$
\Delta_{0}^{\prime}\left[P_{0}-H_{t}^{\prime} P_{1} H_{t}-2\left(g_{0}-H_{t}^{\prime} g_{1}\right) w_{t}+w_{t}^{\prime} \alpha w_{t}\right] \Delta_{0}>0
$$

Condition (6) follows from corollary 1.

\section{GLOBAL ANALYSIS OF PLS}

In the previous section, we showed how a single impact map can be efficiently globally analyzed using SuLF. This section briefly explains how impact maps associated with PLS are combined to globally analyze the system (details are left to Sections VI and VII). There are basically three main steps to achieve this goal: characterization of impact maps, definition of SuLF, and solution of stability conditions.

Step 1) Impact Maps

a) Identification of all impact maps associated with the PLS. If the system has $m$ switching surfaces then there are at most $2 m^{2}$ impact maps. The actual number of impact maps required to analyze the system is typically smaller due to certain properties of a system like symmetries or just the fact that not all switches are possible.

b) In order to reduce conservatism, it is important to characterize the domain of each impact map, as explained in Section VIII.A. Impact maps that have an empty domain do not need to be further considered, neither those points in switching surfaces that converge asymptotically to an equilibrium point without switching (see the middle of Fig. 3). Also, certain necessary conditions must be checked to guarantee that a trajectory, starting in a switching surface, does not grow unbounded without switching (as in the left of Fig. 3).

c) For each impact map find the set of switching times $\mathcal{T}$.

d) For each impact map, find an $x_{0}^{*}$ belonging to the hyperplane where the domain of an impact is defined, such that the assumption of Theorem 1 is satisfied. If this is not possible, characterize the switching times where the assumption is not satisfied and then proceed as explained in Section III.

Step 2) SuLF

a) Define all SuLF on the respective domains of impact maps.

b) Characterize constraints on SuLF related with continuity across boundaries, and with equilibrium points or limit cycles that belong to or intersect the domain of impact maps.
Step 3) Stability Conditions

a) For each impact map, Theorem 2 provides stability conditions (6) that can be relaxed to LMIs like (8). The stability conditions must then be solved simultaneously to find the parameters of the SuLF.

b) Bounds on switching times. For many impact maps, it is sufficient to check the associated stability condition (6) on a bounded subset of switching times $\mathcal{T}$, instead of all $t \in \mathcal{T}$.

c) Improvement of stability conditions. If the LMIs provided by Corollary 3 fail to find a feasible solution then less conservative conditions can be used, as explained in Section VIII.

d) An alternative to solve the above set of LMIs is to consecutively add new LMI's until the stability conditions are satisfied, since checking an LMI is much easier than solving it. The following algorithm can be used instead.

i) Initialize the SuLF with some parameters. The set of LMIs is an empty set at this time.

ii) Check if the stability conditions are satisfied for all switching times (or switching times bounds).

iii) If not, take a switching time where it was not satisfied and add a new LMI to the set of LMIs. Solve this new set of LMIs, get new parameters for the SuLF, and go back to ii). If yes, the algorithm ends.

To better understand each of the above steps in analyzing PLS with SuLF, several classes of PLS are considered. Each of these classes was carefully chosen to separately deal with different issues in each step of the algorithm, and to illustrate with examples the efficiency of this new methodology. By increasing complexity, we first analyzed limit cycles of relay feedback systems (RFS) [3]. For symmetric unimodal limit cycles there is only a single impact map that needs to be studied. This means that global analysis of symmetric unimodal limit cycles of RFS follows directly from Theorem 2.

Then, Section VI analyzes on/off systems to explain how this new methodology is used to globally analyze equilibrium points, and how more than one impact map is simultaneously analyzed. Finally, Section VII considers saturation systems to show how to deal with multiple switching surfaces. The success in globally analyzing a large number of examples of these classes of PLS demonstrated the potential of these new results in globally analyzing other, more complex classes of PLS using a combination of the ideas discussed above.

\section{ON-OFF SYSTEMS (OFS)}

This section addresses the problem of global stability analysis of OFS. An OFS can be thought of as an LTI system that switches between closed (on) and open (off) loop, or as a lower 
bound saturation. OFS can be found in many biological and engineering applications. In biology, concentrations of substrates have a lower bound saturation since they must always be positive. In electronic circuits, diodes can be approximated by on/off nonlinearities. Also, transient behavior of logical circuits that involve latches/flip-flops performing on/off switching can be modeled with on/off circuits and saturations. In aircraft control [1], a max controller was designed to achieve good tracking of the pilot's input without violating safety margins.

As in RFS, a large number of examples is successfully proven globally stable, including systems with unstable subsystems, systems of relative degree larger than one and of high dimension, and systems with unstable nonlinearity sectors, for which classical methods like small gain theorem, Popov criterion, Zames-Falb criterion [4], and integral quadratic constraints [5]-[8], fail to analyze. In fact, it is still an open problem whether there exists an example with a globally stable equilibrium point that could not be successfully analyzed with this new methodology.

\section{A. Problem Formulation}

This section starts by defining OFS and giving some necessary conditions for the global stability of a unique locally stable equilibrium point. Consider a SISO LTI system

$$
\left\{\begin{array}{l}
\dot{x}=A x+B u \\
y=C x
\end{array}\right.
$$

where $x \in \mathbb{R}^{n}$, in feedback with an on/off controller (see Fig. 7) given by

$$
u(t)=\max \{0, y(t)-d\}
$$

where $d \in \mathbb{R}$. By a solution of (9) and (10), we mean functions $(x, y, u)$ satisfying (9) and (10). Since $u$ is continuous and globally Lipschitz, $A x+B u$ is also globally Lipschitz. Thus, the OFS has a unique solution for any initial state.

In the state-space, the on/off controller introduces a switching surface composed of an hyperplane of dimension $n-1$ given by

$$
S=\left\{x \in \mathbb{R}^{n}: C x=d\right\} .
$$

On one side of the switching surface $(C x<d)$, the system is given by $\dot{x}=A x$. On the other side $(C x>d)$, the system is given by $\dot{x}=A x+B(C x-d)=A_{1} x+B_{1}$, where $A_{1}=$ $A+B C$ and $B_{1}=-B d$. The vector field is continuous along the switching surface since $A_{1} x+B_{1}=A x$ for any $x \in S$.

OFS have either zero, one, two, or a continuum of nonisolated equilibrium points. To be globally stable, an OFS needs to have a unique locally stable equilibrium point. Next are necessary conditions for the existence of a single locally stable equilibrium point for different values of $d$.

If $d>0$ there is at least one equilibrium point at the origin. In this case, it is necessary that $A$ is Hurwitz to guarantee the origin is locally stable. If $A_{1}$ is invertible, the subsystem $\dot{x}=A_{1} x+B_{1}$ has an equilibrium point at $-A_{1}^{-1} B_{1}$. To guarantee the OFS has only one equilibrium point at the origin, it is necessary that $-C A_{1}^{-1} B_{1}<d$. It is also necessary that $A_{1}$ has no real unstable

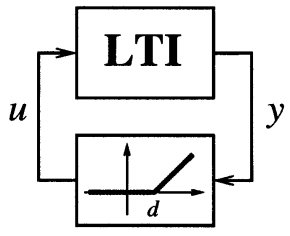

Fig. 7. OFS.

eigenvalues or, otherwise, the system will have trajectories that grow unbounded (with possible exceptions occurring when an eigenvector associated with an unstable real eigenvalue is perpendicular to $C^{\prime}$ ).

When $d=0$, the origin is the only equilibrium point. For the same reasons as before, it is necessary that both $A$ and $A_{1}$ do not have real unstable eigenvalues. In this case, there is no "easy" way to check if the origin is locally stable.

When $d<0$, it must be true that $A_{1}$ is Hurwitz and $A$ has no real unstable poles. It is also necessary that $-C A_{1}^{-1} B_{1}>d$ or otherwise the system will have no equilibrium points.

Assume, without loss of generality, that $d \geq 0$. If $d<0$ and all necessary conditions are met, with an appropriate change of variables $\left(x_{\text {new }}=-x-A_{1}^{-1} B_{1}\right)$ the problem can be transformed to one of analyzing the origin with $d_{\text {new }} \geq 0$. In this case, $A_{\text {new }}=A_{1}, A_{1 \text { new }}=A, B_{1 \text { new }}=A A_{1}^{-1} B_{1}$, and $d_{\text {new }}=$ $-d-C A_{1}^{-1} B_{1} \geq 0$.

Define $S_{+}=\{x \in S: C A x \geq 0\}$, the set of points in $S$ that can be reached by trajectories starting at any $x_{0}$ such that $C x_{0}<d$ (see the left of Fig. 8). Similarly, define $S_{-}=\{x \in$ $S: C A x \leq 0\}$. Note that $S=S_{+} \bigcup S_{-}$.

From here on, assume $d>0$. In terms of stability analysis, $d=0$ is a special case of when $d>0$, and will be considered separately in Section VI.E.

Since $A$ is Hurwitz, there exists an $S^{*} \subset S_{-}$such that any trajectory starting in that set does not switch again and converges asymptotically to the origin. In other words, $x_{0} \in S^{*}$ if and only if $C e^{A t} x_{0}=d$ does not have a solution for all $t>0$. Note that $S^{*}$ is not empty. To see this, let $P>0$ satisfy $P A+A^{\prime} P=-I$. Then, an obvious point in $S^{*}$ is the point $x_{1}^{*}$ obtained from the intersection of $S$ with the level set $x^{\prime} P x=k$, where $k \geq 0$ is chosen such that the ellipse $x^{\prime} P x=k$ is tangent to $S$ (see the right-hand side of Fig. 8).

\section{B. Impact Maps for OFS}

Consider a trajectory $x(t)$ starting at some point $x_{0} \in S_{+}$ (see Fig. 9). If all necessary conditions are met, $x(t)$ eventually switches at $x_{1} \in S_{-}$. If $x_{1} \in S^{*}$, the trajectory converges asymptotically to the origin without switching. If $x_{1} \in S_{-} \backslash S^{*}$, the trajectory switches again at $x_{2} \in S_{+}$, and then at $x_{3}$, and so on. The idea is to check if the sequence $x_{1}, x_{3}, \ldots$ is getting closer and eventually enter $S^{*}$. If so, then $x_{2 N-1} \in S^{*}$ for some large enough $N$, and prove the origin is globally asymptotically stable.

Let $x_{0}=x_{0}^{*}+\Delta_{0}, x_{1}=x_{1}^{*}+\Delta_{1}$, and $x_{2}=x_{0}^{*}+\Delta_{2}$, where $x_{0}^{*}, x_{1}^{*} \in S$, and $C \Delta_{i}=0$. Define $x_{0}^{*}(t)\left(x_{1}^{*}(t)\right)$ as the trajectory of $\dot{x}=A_{1} x+B_{1}(\dot{x}=A x)$, starting at $x_{0}^{*}\left(x_{1}^{*}\right)$, for all $t \geq 0$. Since $x_{i}^{*}$ can be any points in $S$, choose them such that $C x_{i}^{*}(t)<d$ for all $t>0$. As explained in Appendix I.A, this is 

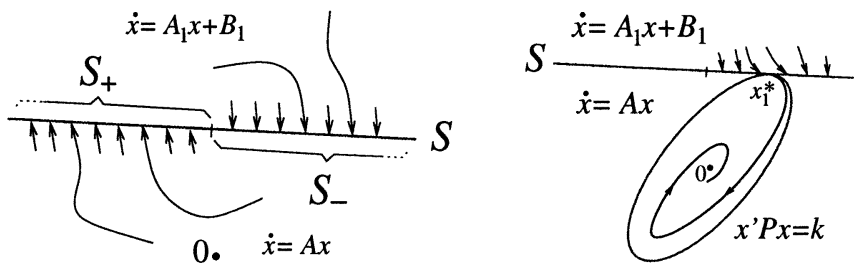

Fig. 8. Both sets $S_{+}$and $S_{-}$in $S$ (left). How to obtain $x_{1}^{*}$ (right).

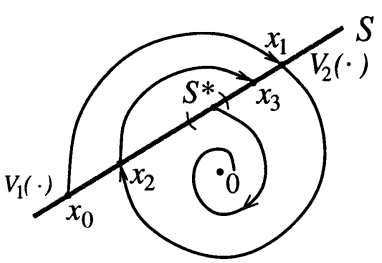

Fig. 9. Possible trajectories for an OFS.

always possible, even when $A_{1}$ is unstable (as long as it has at least one stable eigenvalue with an associated eigenvector that is not perpendicular to $C^{\prime}$ ). The reason for this particular choice of $x_{0}^{*}$ and $x_{1}^{*}$ is so that $C x_{i}^{*}(t)-d \neq 0$ for all $t>0$. This will be necessary in proposition 1 .

There are two impact maps of interest associated with an OFS. Impact map 1 takes points from $S_{+}$(the departure set of impact map 1) and maps them into $S_{-}$(the associated arrival set). Impact map 2 takes points from $S_{-} \backslash S^{*}$ (the departure set of impact map 2) and maps them back to $S_{+}$(the associated arrival set). Note that $S^{*}$ does not belong to the domain of either impact map since every trajectory starting in $S^{*}$ does not switch again.

As in definition 1, define the sets of switching times $\mathcal{T}_{1}$ and $\mathcal{T}_{2}$ associated with the respective impact maps. For each $t_{1} \in \mathcal{T}_{1}$ and $t_{2} \in \mathcal{T}_{2}$ define also $S_{t_{1}} \subset S_{+}$and $S_{t_{2}} \subset S_{-} \backslash S^{*}$ similarly as $S_{t}$ was defined in Section III. For instance, for impact map 1, $S_{t_{1}}$ is the set of points in $S_{+}$that have an associated switching time $t_{1}$, i.e., $S_{t_{1}}$ is the set of all initial conditions $x_{0} \in S_{+}$such that $C x(t) \geq d$ on $\left[0, t_{1}\right]$, and $C x\left(t_{1}\right)=d$. Note that both $S_{t_{1}}$ and $S_{t_{2}}$ are subsets of linear manifolds of dimension $n-2$.

\section{Global Asymptotic Stability of OFS}

Before presenting the main result of this section, Theorem 1 is used to show that each impact map associated with OFS can be represented as a linear transformation analytically parametrized by the correspondent switching time.

Proposition 1: Define

$$
w_{1}(t)=\frac{C e^{A_{1} t}}{d-C x_{0}^{*}(t)} \quad \text { and } w_{2}(t)=\frac{C e^{A t}}{d-C x_{1}^{*}(t)}
$$

Let $H_{1}(t)=e^{A_{1} t}+\left(x_{0}^{*}(t)-x_{1}^{*}\right) w_{1}(t)$ and $H_{2}(t)=e^{A t}+$ $\left(x_{1}^{*}(t)-x_{0}^{*}\right) w_{2}(t)$. Then, for any $\Delta_{0} \in S_{+}-x_{0}^{*}$ there exists a $t_{1} \in \mathcal{T}_{1}$ such that $\Delta_{1}=H_{1}\left(t_{1}\right) \Delta_{0}$. Such $t_{1}$ is the switching time associated with $\Delta_{1}$. Similarly, for any $\Delta_{1} \in S_{-} \backslash S^{*}-x_{1}^{*}$ there exists a $t_{2} \in \mathcal{T}_{2}$ such that $\Delta_{2}=H_{2}\left(t_{2}\right) \Delta_{1}$. Such $t_{2}$ is the switching time associated with $\Delta_{2}$.
Next, define two SuLF $V_{1}$ and $V_{2}$ on $S_{+}$and $S_{-} \backslash S^{*}$, respectively. Global asymptotically stability of the origin follows if both impact maps are simultaneously contracting

$$
\begin{array}{ll}
V_{2}\left(\Delta_{1}\right)<V_{1}\left(\Delta_{0}\right) & \text { for all } \Delta_{0} \in S_{+}-x_{0}^{*} \\
V_{1}\left(\Delta_{2}\right)<V_{2}\left(\Delta_{1}\right) & \text { for all } \Delta_{1} \in S_{-} \backslash S^{*}-x_{1}^{*} .
\end{array}
$$

If $V_{i}$ are chosen quadratic as in (7), the next theorem extends Corollary 2 for the case where we simultaneously prove contraction of two impact maps. As a short hand, let $H_{i t}=H_{i}(t)$ and $w_{i t}=w_{i}(t)$.

Theorem 3: Let $\alpha=\alpha_{1}-\alpha_{2}$. Define

$$
\begin{aligned}
R_{1}(t)= & P_{1}-H_{1 t}^{\prime} P_{2} H_{1 t} \\
& -2\left(g_{1}-H_{1 t}^{\prime} g_{2}\right) w_{1 t}+w_{1 t}^{\prime} \alpha w_{1 t} \\
R_{2}(t)= & P_{2}-H_{2 t}^{\prime} P_{1} H_{2 t} \\
& -2\left(g_{2}-H_{2 t}^{\prime} g_{1}\right) w_{2 t}-w_{2 t}^{\prime} \alpha w_{2 t} .
\end{aligned}
$$

The origin of the OFS is globally asymptotically stable if there exist $P_{1}, P_{2}>0$, and $g_{1}, g_{2}, \alpha$ such that

$$
\begin{cases}R_{1}\left(t_{1}\right)>0 & \text { on } S_{t_{1}}-x_{0}^{*} \\ R_{2}\left(t_{2}\right)>0 & \text { on } S_{t_{2}}-x_{1}^{*}\end{cases}
$$

for all switching times $t_{1} \in \mathcal{T}_{1}$ and $t_{2} \in \mathcal{T}_{2}$.

As in Corollary 3, a relaxation on $S_{t_{1}}$ and $S_{t_{2}}$ results in computationally efficient conditions.

Corollary 4: The origin of the OFS is globally asymptotically stable if there exist $P_{1}, P_{2}>0$ and $g_{1}, g_{2}, \alpha$ such that

$$
\begin{cases}R_{1}\left(t_{1}\right)>0 & \text { on } S-x_{0}^{*} \\ R_{2}\left(t_{2}\right)>0 & \text { on } S-x_{1}^{*}\end{cases}
$$

for all switching times $t_{1} \in \mathcal{T}_{1}$ and $t_{2} \in \mathcal{T}_{2}$.

For each $t_{1}, t_{2}$ these conditions are LMIs which can be solved for $P_{1}, P_{2}>0$ and $g_{1}, g_{2}, \alpha$ using efficient available software. As shown next, although conditions (14) are more conservative than (13), they are enough to prove global asymptotic stability of many important OFS. Section VIII, and, in particular, Section VIII-C, explains how to approximate conditions (13) with less conservative sets of LMIs than (14).

Each condition in (14) depends only on a single scalar parameter, i.e., $R_{1}$ depends only on $t_{1}$ and $R_{2}$ depends only on $t_{2}$. Thus, each condition is an infinite dimensional LMI that can be gridded independently to obtain two finite sets of LMIs. A less conservative condition, of the form $R\left(t_{1}, t_{2}\right)>0$, could be obtained (representing the contraction of the map from $x_{1}$ to $x_{3}$ in Fig. 9). Obtaining a finite set of LMIs from $R\left(t_{1}, t_{2}\right)>0$, however, involves griding the two-dimensional set $\mathcal{T}_{1} \times \mathcal{T}_{2}$, resulting in a much larger number of LMIs. The problem would easily become computationally intractable, especially when analyzing other, more complex PLS that may require the analysis of a large number of impact maps.

\section{Examples}

Software code has been written in MATLAB to analyze OFS, where the latest version is available at [15]. The user supplies a transfer function of an LTI system and the displacement of the nonlinearity switch $d$. If the OFS is proven globally stable, the software returns the parameters of the SuLF $V_{1}$ and $V_{2}$ in (11), 
(12). The MATLAB function also plots the minimum eigenvalues of each $R_{i}\left(t_{i}\right)$ in (14) on a finite subset of $\mathcal{T}_{i}$. Bounds on maximum switching times are considered in Appendix I.C. Also, certain necessary conditions imposed at $t_{i}=0$ are discussed in Appendix I-B.

Example 6.1: Consider the OFS on the left-hand side of Fig. 10 with $d=1$. The origin of this system is locally stable.

Using conditions (14), the origin is also proven globally asymptotically stable. The right side of Fig. 10 shows the minimum eigenvalue of each condition (14), which are positive on their respective sets of switching times $\mathcal{T}_{1}=(0,1.85)$ and $\mathcal{T}_{2}=(0,4.7)$.

An interesting fact about this system is that it has an unstable nonlinearity sector. If the on/off nonlinearity is replaced by a linear constant gain of $1 / 2$, the system becomes unstable (Fig. 11). Thus, classical analysis tools like small gain theorem, Popov or Zames-Falb criterion, and integral quadratic constraints, would all fail to analyze OFS of this nature.

Example 6.2: Consider the OFS in Fig. 12 with $d=1$ and $k>0$. The origin is locally stable for any $k>0$.

Since $\left\|C e^{A t} B\right\|_{\mathcal{L}_{1}}=k$, the small gain theorem can be applied whenever $k \leq 1$. When $k>1$, however, the small gain theorem fails to analyze the system. Let $k=2$. Using conditions (14), the origin is proven globally asymptotically stable. The right side of Fig. 12 confirms that conditions (14) are satisfied in some intervals $\left(0, t_{i \max }\right), i=1,2$, which are bounds on the sets of switching times (see Appendix I-C).

Example 6.3: Consider the OFS in Fig. 13 with $d=1$. The origin is locally stable. $A_{1}$, however, is unstable.

Even if $A_{1}$ is unstable, for third-order systems it is easy to find the sets of switching times since the switching surface has dimension 2 . In this case, $\mathcal{T}_{1}=(0,21.8)$ and $\mathcal{T}_{2}=(0,4.2)$. Although $A_{1}$ is unstable, using conditions (14) the origin is proven globally asymptotically stable. The right-hand side of Fig. 13 shows how conditions (14) are satisfied on the respective sets of switching times.

\section{E. Special Case: $d=0$}

When $d=0$, the stability conditions can be relaxed to a set of LMIs that are, in general, much less conservative than conditions (14) and even conditions (27). First, since the origin belongs to both subsystems $\dot{x}=A x$ and $\dot{x}=(A+B C) x$, it is only required that both systems do not have real unstable poles. Also, $d=0$ means $x_{0}^{*}=x_{1}^{*}=g_{1}=g_{2}=0$ and $\alpha=0$.

The impact maps reduce to $\Delta_{1}=e^{A_{1} t_{1}} \Delta_{0}$ and $\Delta_{2}=e^{A t_{2}} \Delta_{1}$. Thus, the stability conditions are simply

$$
\begin{array}{cc}
\Delta_{1}^{\prime} P_{2} \Delta_{1} & <\Delta_{0}^{\prime} P_{1} \Delta_{0} \\
\Delta_{2}^{\prime} P_{1} \Delta_{2} & <\Delta_{1}^{\prime} P_{2} \Delta_{1}
\end{array} \Leftrightarrow \begin{gathered}
\Delta_{0}^{\prime}\left(P_{1}-e^{A_{1}^{\prime} t_{1}} P_{2} e^{A_{1} t_{1}}\right) \Delta_{0}>0 \\
\Delta_{1}^{\prime}\left(P_{2}-e^{A^{\prime} t_{2}} P_{1} e^{A t_{2}}\right) \Delta_{1}>0
\end{gathered}
$$

for some $P_{1}, P_{2}>0$, all $\Delta_{0} \in S_{t_{1}}, \Delta_{1} \in S_{t_{2}}$, and all switching times $t_{1} \in \mathcal{T}_{1}, t_{2} \in \mathcal{T}_{2}$. Since $C \Delta_{0}=C \Delta_{1}=C \Delta_{2}=$ 0, $C e^{A_{1} t_{1}} \Delta_{0}=C e^{A t_{2}} \Delta_{1}=0$. That is, for fixed values of $t_{1}$ and $t_{2}, \Delta_{0}$ and $\Delta_{1}$ are restricted to a subspace of dimension $n-2$. Let $\Pi \in C^{\perp}$, where $C^{\perp}$ are the orthogonal complements to $C$, i.e., matrices with a maximal number of column vectors forming an orthonormal set such that $C C^{\perp}=0$. Define $l_{t_{1}} \in\left(C e^{A_{1} t_{1}} \Pi\right)^{\perp}$ and $l_{t_{2}} \in\left(C e^{A t_{2}} \Pi\right)^{\perp}$.
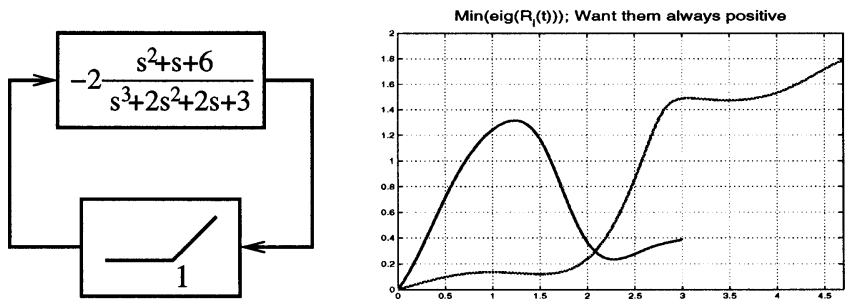

Fig. 10. 3rd-order system with unstable nonlinearity sector.

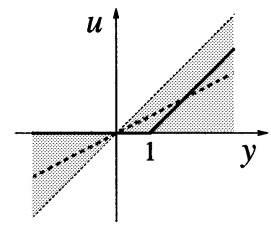

Fig. 11. Sector (shaded) of on/off controller and constant gain of $(1 / 2)$ (dashed).
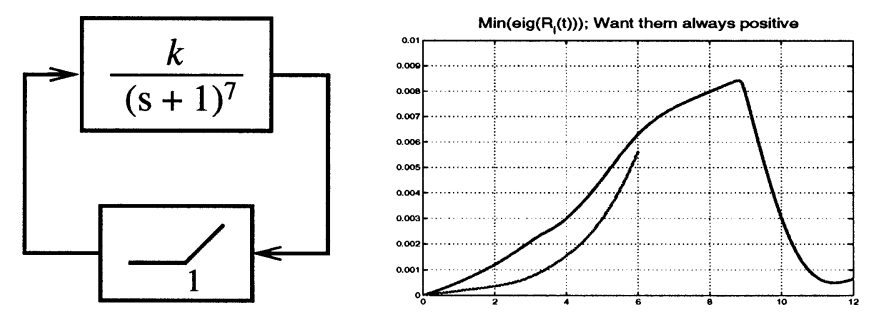

Fig. 12. System with relative degree 7 (left). Global stability analysis for $k=$ 2 (right).
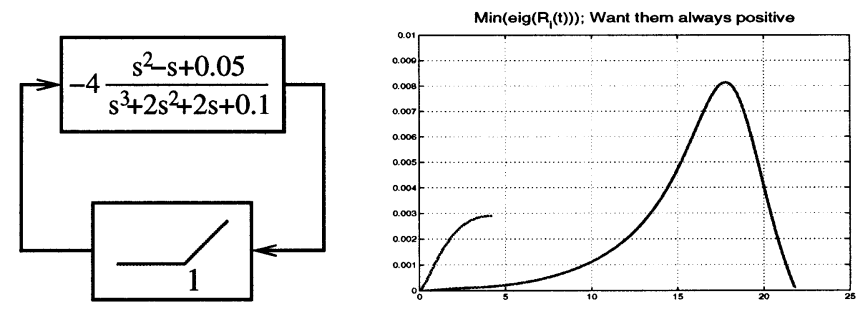

Fig. 13. System with unstable $A_{1}$.

Theorem 4: The origin of the OFS with $d=0$ is globally asymptotically stable if there exist $P_{1}, P_{2}>0$ such that

$$
\begin{cases}l_{t_{1}}^{\prime} \Pi^{\prime}\left(P_{1}-e^{A_{1}^{\prime} t_{1}} P_{2} e^{A_{1} t_{1}}\right) \Pi l_{t_{1}} & >0 \\ l_{t_{2}}^{\prime} \Pi^{\prime}\left(P_{2}-e^{A^{\prime} t_{2}} P_{1} e^{A t_{2}}\right) \Pi l_{t_{2}} & >0\end{cases}
$$

for all switching times $t_{1} \in \mathcal{T}_{1}$ and $t_{2} \in \mathcal{T}_{2}$.

\section{SATURATION SYSTEMS}

In the state-space, OFS are divided in two partitions by a single switching surface. This section shows how impact maps and SuLF are used to globally analyze PLS with more than two state-space partitions and more than one switching surface. To demonstrate these ideas, a class of PLS known as saturation systems (SAT) is analyzed. Saturations are present in most biological and engineering systems, like in bounds of concentrations or in available power supply of actuators. 
It is well known that linear feedback laws when saturated can lead to instability. The problem of stabilizing linear systems with bounded controls has been extensively studied. See, for example, [16]-[18] and the references therein.

For analysis of SAT, the Popov criterion is simple but conservative. The Zames-Falb criterion [4] can be used when the nonlinearity's slope is restricted, but the method is difficult to implement. IQC-based analysis [5]-[8] gives conditions in the form of LMIs that, when satisfied, guarantee stability of SAT. However, none of these analysis tools can be used when a SAT has an unstable nonlinearity sector.

As in OFS, a large number of examples was successfully proven globally stable, including high-order systems, systems of relative degree larger than one, and systems with unstable nonlinearity sectors for which all classical methods fail to analyze. In fact, existence of an example with a globally stable equilibrium point that could not be successfully analyzed with this new methodology is still an open problem.

\section{A. Problem Formulation}

This section starts by defining SAT followed by some necessary conditions for global stability of a unique locally stable equilibrium point. Consider a SISO LTI system (9) in feedback with a saturation controller (see Fig. 14) given by

$$
u(t)= \begin{cases}-d & \text { if } y(t)<-d \\ y(t) & \text { if }|y(t)| \leq d \\ d & \text { if } y(t)>d\end{cases}
$$

where $d>0$ (if $d=0$ the system is simply linear). By a solution of (9) and (16) we mean functions $(x, y, u)$ satisfying (9) and (16). The SAT has a unique solution for any initial state since $A x+B u$ is globally Lipschitz.

In the state-space, the saturation controller introduces two switching surfaces composed of hyperplanes of dimension $n-1$ given by

$$
S=\left\{x \in \mathbb{R}^{n}: C x=d\right\}
$$

and

$$
\underline{S}=-S=\left\{x \in \mathbb{R}^{n}: C x=-d\right\} .
$$

On one side of the switching surface $S(C x>d)$, the system is governed by $\dot{x}=A x+B d$. In between the two switching surfaces $(|C x| \leq d), \dot{x}=A x+B C x=A_{1} x$, where $A_{1}=$ $A+B C$. Finally, on the other side of $\underline{S}(C x<-d), \dot{x}=$ $A x-B d$. The vector field (9), (16) is continuous along the switching surfaces since $A x+B d=A_{1} x$ for any $x \in S$, and $A_{1} x=A x-B d$ for any $x \in \underline{S}$.

SAT can exhibit extremely complex behaviors. Some may be chaotic, others have one, three, or a continuum of equilibrium points, limit cycles, or even some combination of all these behaviors. We are interested in those SAT with a unique locally stable equilibrium point. For that, it is necessary that $A_{1}$ is Hurwitz and, if $A$ is invertible, that $-C A^{-1} B<1$, so the origin is the only equilibrium point. It is also necessary that $A$ has no eigenvalues with positive real part, or otherwise there are initial conditions for which the system will grow unbounded (see, for example, [19]).

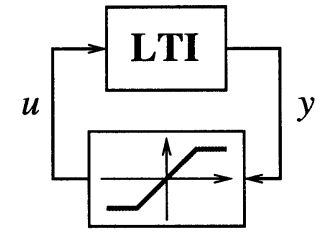

Fig. 14. Saturation system.

As in OFS, define $S_{+}=\left\{x \in S: C A_{1} x \geq 0\right\}, S_{-}=\{x \in$ $\left.S: C A_{1} x \leq 0\right\}$, and also $\underline{S}_{+}=-S_{+}$and $\underline{S}_{-}=-S_{-}$. Since $A_{1}$ must be Hurwitz, there is a nonempty subset of $S_{-}$such that any trajectory starting in that set will not switch again and will converge asymptotically to the origin. In other words, let $S^{*} \subset S_{-}$be the set of points $x_{0}$ such that $C e^{A_{1} t} x_{0}= \pm d$ do not have a solution for any $t>0$.

\section{B. Impact Maps for Saturation Systems}

The strategy of the proof is similar to OFS. The main difference is that a trajectory starting in $S_{-} \backslash S^{*}$ can also switch at $\underline{S}$ (see Fig. 15). Let $S_{d}\left(S_{-d}\right)$ be the set of points in $S_{-} \backslash S^{*}$ that will switch in $S(\underline{S})$. If $x_{1} \in S_{d}\left(x_{1} \in S_{-d}\right)$ the trajectory switches at $x_{2 a} \in S_{+}\left(x_{2 b} \in \underline{S}_{+}\right)$, and then again at $x_{3 a}\left(x_{3 b}\right)$, and so on. The idea is to check if the sequence $x_{1}, x_{3 a} /-x_{3 b}, \ldots$ is getting closer and eventually enter $S^{*}$. If so, then $\pm x\left(t_{2 N-1}\right) \in S^{*}$ for some large enough $N$, and prove the origin is globally asymptotically stable.

Let $x_{0}=x_{0}^{*}+\Delta_{0}, x_{1}=x_{1}^{*}+\Delta_{1}, x_{2 a}=x_{0}^{*}+\Delta_{2 a}$, and $x_{2 b}=-x_{0}^{*}+\Delta_{2 b}$, where $x_{0}^{*}, x_{1}^{*} \in S$ and $C \Delta_{i}=0$. Define $x_{0}^{*}(t)\left(x_{1}^{*}(t)\right)$ as the trajectory of $\dot{x}=A x+B d\left(\dot{x}=A_{1} x\right)$, starting at $x_{0}^{*}\left(x_{1}^{*}\right)$, for all $t \geq 0$. Since $x_{i}^{*}$ are any points in $S$, they are chosen such that $C x_{i}^{*}(t)<d$ for all $t>0$. This choice of $x_{0}^{*}$ and $x_{1}^{*}$ is always possible. $x_{1}^{*}$ is found as explained in OFS. In this case, $x_{1}^{*} \in S^{*}$ is given by

$$
x_{1}^{*}=\frac{P_{d}^{-1} C^{\prime}}{C P_{d}^{-1} C^{\prime}} d
$$

where $P_{d}>0$ satisfies $P_{d} A_{1}+A_{1}^{\prime} P_{d}=-I$. Similarly, when $A$ is a stable matrix $x_{0}^{*}$ is given by

$$
x_{0}^{*}=\left(d+c A^{-1} B d\right) \frac{P_{u}^{-1} C^{\prime}}{C P_{u}^{-1} C^{\prime}}-A^{-1} B d
$$

where $P_{u}>0$ satisfies $P_{u} A+A^{\prime} P_{u}=-I$. If $A$ is not stable, $x_{0}^{*}$ is found as in Appendix I-A.

Just like RFS, SAT are symmetric around the origin. Thus, for analysis purposes, it is equivalent to consider the trajectory starting at $x_{2 b}$ or $-x_{2 b}$ (see Fig. 15). This means there are only three impact maps that need to be analyzed. Impact map 1 takes points from $S_{+}$and maps them into $S_{-}$. Impact map $2 a$ takes points from $S_{d}$ and maps them back to $S_{+}$. Finally, impact map $2 b$ takes points from from $S_{-d}$ and maps them into $\underline{S}_{+}$. As before, define the sets of switching times $\mathcal{T}_{1}, \mathcal{T}_{2 a}$, and $\mathcal{T}_{2 b}$. Appendix II shows how to obtain bounds on these sets. Define also $S_{t_{1}}, S_{t_{2 a}}$, and $S_{t_{2 b}}$ as in Sections III and VI-B.

\section{Global Asymptotic Stability of Saturation Systems}

The linear representation of impact maps associated with SAT follows as in Theorem 1 and OFS. 

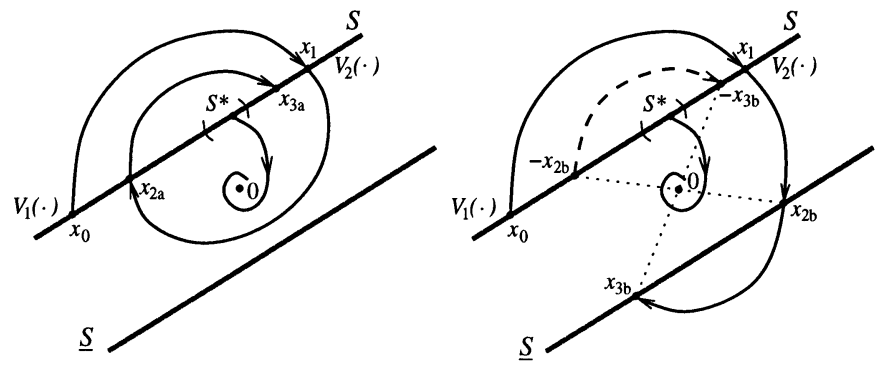

Fig. 15. Possible trajectories for a SAT.

Proposition 2: Define

$$
\begin{aligned}
w_{1 t} & =\frac{C e^{A t}}{d-C x_{0}^{*}(t)}, w_{2 a t}=\frac{C e^{A_{1} t}}{d-C x_{1}^{*}(t)} \\
w_{2 b t} & =\frac{C e^{A_{1} t}}{-d-C x_{1}^{*}(t)}
\end{aligned}
$$

Let $H_{1 t}=e^{A t}+\left(x_{0}(t)^{*}-x_{1}^{*}\right) w_{1 t}, H_{2 a t}=e^{A_{1} t}+\left(x_{1}(t)^{*}-\right.$ $\left.x_{0}^{*}\right) w_{2 a t}$, and $H_{2 b t}=e^{A_{1} t}+\left(x_{1}(t)^{*}+x_{0}^{*}\right) w_{2 b t}$. Then, for any $\Delta_{0} \in S_{+}-x_{0}^{*}, \Delta_{1} \in S_{d}-x_{1}^{*}$, and $\Delta_{1} \in S_{-d}-x_{1}^{*}$, there exist $t_{i} \in \mathcal{T}_{i}, i=\{1,2 a, 2 b\}$, such that

$$
\Delta_{1}=H_{1}\left(t_{1}\right) \Delta_{0}, \Delta_{2 a}=H_{2 a}\left(t_{2 a}\right) \Delta_{1}, \Delta_{2 b}=H_{2 b}\left(t_{2 b}\right) \Delta_{1}
$$

respectively. Such $t_{i}$ are the switching times associated with $\Delta_{i}, i=\{1,2 a, 2 b\}$.

Next, define two SuLF $V_{1}$ and $V_{2}$ on $S_{+}$and $S_{-} \backslash S^{*}$, respectively. Global asymptotically stability of the origin follows if all impact maps are simultaneously contracting

$$
\begin{aligned}
& V_{2}\left(\Delta_{1}\right)<V_{1}\left(\Delta_{0}\right) \text { forall } \Delta_{0} \in S_{+}-x_{0}^{*} \\
& V_{1}\left(\Delta_{2 a}\right)<V_{2}\left(\Delta_{1}\right) \text { forall } \Delta_{1} \in S_{d}-x_{1}^{*} \\
& V_{1}\left(-\Delta_{2 b}\right)<V_{2}\left(\Delta_{1}\right) \text { forall } \Delta_{1} \in S_{-d}-x_{1}^{*} \text {. }
\end{aligned}
$$

In the last inequality, $\Delta_{2 b} \in \underline{S}_{+}+x_{0}^{*}$ was mapped into $-\Delta_{2 b} \in$ $S_{+}-x_{0}^{*}$, taking advantage of the symmetry of the system. If $V_{i}$ are quadratic as in (7), we have the following result.

Theorem 5: Let $v_{i t}=w_{i t}^{\prime} \alpha w_{i t}, i=\{1,2 a, 2 b\}$ and define

$$
\begin{aligned}
R_{1}(t)= & P_{1}-H_{1 t}^{\prime} P_{2} H_{1 t} \\
& -2\left(g_{1}-H_{1 t}^{\prime} g_{2}\right) w_{1 t}+v_{1} \\
R_{2 a}(t)= & P_{2}-H_{2 a t}^{\prime} P_{1} H_{2 a t} \\
& -2\left(g_{2}-H_{2 a t}^{\prime} g_{1}\right) w_{2 a t}-v_{2 a t} \\
R_{2 b}(t)= & P_{2}-H_{2 b t}^{\prime} P_{1} H_{2 b t} \\
& -2\left(g_{2}+H_{2 b t}^{\prime} g_{1}\right) w_{2 b t}-v_{2 b t}
\end{aligned}
$$

where $\alpha=\alpha_{1}-\alpha_{2}$. The origin of the SAT is globally asymptotically stable if there exist $P_{1}, P_{2}>0$ and $g_{1}, g_{2}, \alpha$ such that

$$
\begin{cases}R_{1}\left(t_{1}\right)>0 & \text { on } S_{t_{1}}-x_{0}^{*} \\ R_{2 a}\left(t_{2 a}\right)>0 & \text { on } S_{t_{2 a}}-x_{1}^{*} \\ R_{2 b}\left(t_{2 b}\right)>0 & \text { on } S_{t_{2 b}}-x_{1}^{*}\end{cases}
$$

for all switching times $t_{1} \in \mathcal{T}_{1}, t_{2 a} \in \mathcal{T}_{2 a}$, and $t_{2 b} \in \mathcal{T}_{2 b}$.

As in Corollaries 3 and 4 , a relaxation on $S_{t_{i}}, i=\{1,2 a, 2 b\}$ results in computationally efficient conditions.
Corollary 5: The origin of the SAT is globally asymptotically stable if there exist $P_{1}, P_{2}>0$ and $g_{1}, g_{2}, \alpha$ such that

$$
\begin{cases}R_{1}\left(t_{1}\right)>0 & \text { on } S-x_{0}^{*} \\ R_{2 a}\left(t_{2 a}\right)>0 & \text { on } S-x_{1}^{*} \\ R_{2 b}\left(t_{2 b}\right)>0 & \text { on } S-x_{1}^{*}\end{cases}
$$

for all switching times $t_{1} \in \mathcal{T}_{1}, t_{2 a} \in \mathcal{T}_{2 a}$, and $t_{2 b} \in \mathcal{T}_{2 b}$.

In many cases, conditions (18) and (19) do not need to be satisfied for all switching times. Appendix II shows how bounds on switching times can be obtained when $A$ is Hurwitz. Basically, since $|u| \leq d$ is a bounded input, there exists a bounded set such that any trajectory will eventually enter and stay there. This leads to bounds on the difference between any two consecutive switching times. Conditions (18) and (19) can then be relaxed to be satisfied only on some intervals $\left(t_{i-}, t_{i+}\right)$ instead of all $t_{i} \in \mathcal{T}_{i}$. See Appendix II for details.

\section{Examples}

Software code has been written in MATLAB to analyze SAT, where the latest version is available at [15]. The user supplies a transfer function of an LTI system and the amplitude of the saturation $d$. If the SAT is proven globally stable, the software returns the parameters of the SuLF $V_{1}$ and $V_{2}$ in (17). The mat $l a b$ function also plots the minimum eigenvalues of each $R_{i}\left(t_{i}\right)$ in (19) on bounds of $\mathcal{T}_{i}$.

Example 7.1: Consider the SAT on the left of Fig. 16 with $d=1$. The origin of this system is locally stable.

Using conditions (19), the origin is also proven globally asymptotically stable. The right side of Fig. 16 shows the minimum eigenvalue of each condition (19), which are positive on their respective sets of switching times $\mathcal{T}_{1}=(0,3), \mathcal{T}_{2 a}=(0,3.3)$, and $\mathcal{T}_{2 b}=(0,3.1)$.

As in Example 6.1, this system also has an unstable nonlinearity sector. If the saturation is replaced by a linear constant gain of $1 / 2$, the system becomes unstable (see Fig. 17). Thus, classical analysis tools would again fail to analyze SAT of this nature.

Example 7.2: Consider the SAT in Fig. 18 with $d=1$ and $k>0$. The origin is locally stable for any $k>0$.

As seen in example 6.2, $\left\|C e^{A t} B\right\|_{\mathcal{L}_{1}}=k$ and the small gain theorem can only be applied when $k<1$. Let $k=2$. Using conditions (19), the origin is proven globally asymptotically stable. The right-hand side of Fig. 18 shows how conditions (19) are satisfied on some bounded sets of switching times. For details on how to find these bounds see Appendix II.

Example 7.3: Consider the SAT in Fig. 19 with $d=1$. Popov criterion (see, for example, [13, pp. 419-420]) can easily show that this system is globally asymptotically stable. What is interesting about this system is that it is not exponentially stable. Thus, piecewise quadratic Lyapunov functions [2] fails to analyze the system.

$\mathrm{SuLF}$, however, can analyze and prove global asymptotic stability. The right-hand side of Fig. 19 shows how conditions (19) are satisfied in some intervals $\left(t_{i-}, t_{i+}\right), i=1,2 a, 2 b$. These intervals cannot be found as before since $A$ is not Hurwitz. Alternatively, the analysis must be done for all $t_{i} \geq 0$. The idea of proof is as follows. Analysis near the origin is done as in Appendix I-B. For large values of $t_{i}$, analysis of impact maps $2 a$ 

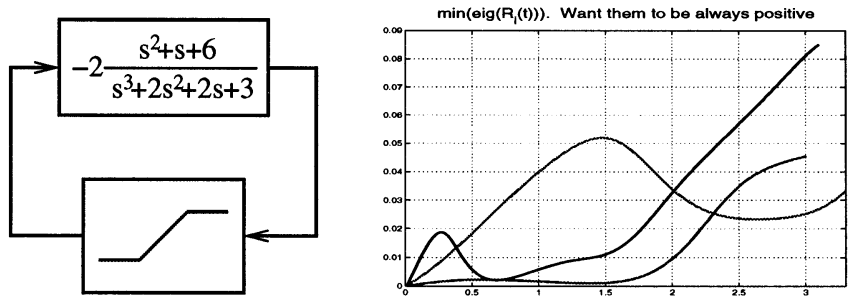

Fig. 16. Third-order system with unstable nonlinearity sector.

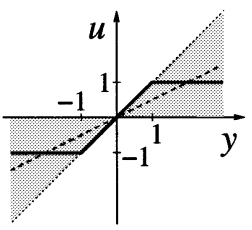

Fig. 17. Sector (shaded) of a saturation and constant gain of $(1 / 2)$ (dashed).
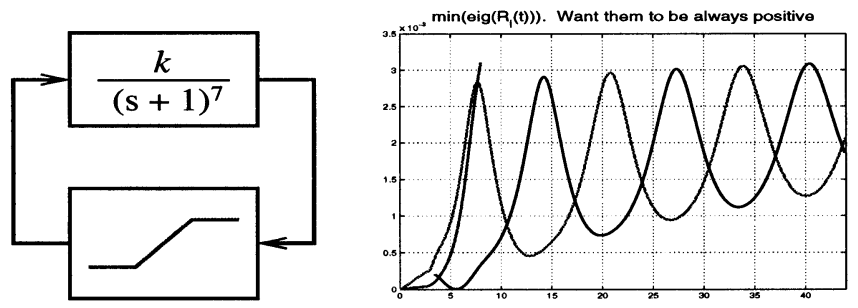

Fig. 18. System with relative degree 7 (left). Global stability analysis when $k=2$ (right).
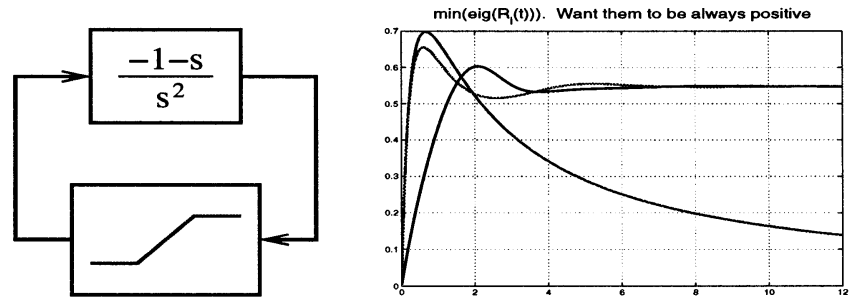

Fig. 19. Second-order system not exponentially stable.

and $2 b$ can be done as in Appendix I-C since the matrix $A_{1}$ is stable. For impact map 1, the constraint $P_{1}=P_{2}$ implies that $R_{1}(\infty)=0$, which is a requirement since the system is not exponentially stable. For a large enough $t_{1+}$, it can be shown that $R_{1}(t)>0$ for all $t \geq t_{1+}$ by proving that $\dot{R}_{1}(t)<0$ for all $t \geq t_{1+}$.

\section{IMPROVEMENT OF STABILITY CONDITIONS}

It is possible to improve condition (8) and, consequently, conditions (14) and (19), at a cost of increased computations. This section explains how to approximate condition (6) with a less conservative set of LMIs than (8).

\section{A. Meaning of Condition (6)}

As seen in several examples in Sections VI and VII, although condition (8) is more conservative than (6), this is enough to prove global asymptotic stability of many important systems. There are, however, examples where (8) fails to prove stability [3]. Condition (8) only takes into account that $\Delta_{0} \in S_{0}-x_{0}^{*}$

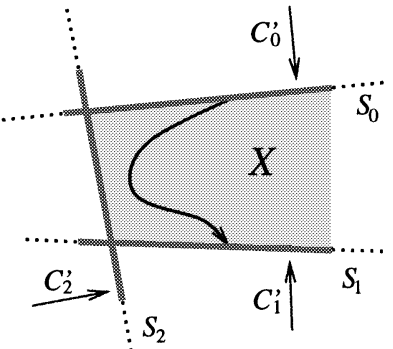

Fig. 20. Trajectories starting at $S_{0}$ must remain in $X$.

(or, equivalently, that $C_{0} \Delta_{0}=0$ ), independently of the value of $t \in \mathcal{T}$. Condition (6), however, uses the information that, for a given $t \in \mathcal{T}, \Delta_{0} \in S_{t}-x_{0}^{*}$. Note that $S_{t} \subset S_{0}$ has one dimension less than $S_{0}$.

The main difficulty with condition (6) is that the set $S_{t}$ is not, in general, easily characterized. Remember that $S_{t}$ is the set of points in $S_{0}^{d}$ that has the same switching time $t$. In other words, a trajectory starting at $\Delta_{0} \in S_{t}-x_{0}^{*}$ satisfies both

$$
\left\{\begin{array}{l}
C_{1} e^{A t} \Delta_{0}=d_{1}-C_{1} x_{0}^{*}(t) \\
x(\tau) \in \bar{X} \quad \forall \tau \in[0, t] .
\end{array}\right.
$$

The equality arises from the fact that $\Delta_{1} \in S_{1}-x_{1}^{*}$, or $C_{1} \Delta_{1}=$ 0 , and is the same as (3). It automatically excludes points in $S_{0}$ that do not intersect $S_{1}$, since such points do not have a finite solution $t>0$ satisfying (3). Note that (3) depends on $t \in \mathcal{T}$, contrasting with $C_{0} \Delta_{0}=0$, which is independent of $t$.

The inclusion in (20) ensures that a trajectory $x(\tau)$, starting at some point in $S_{0}$, stays in the closure of $X$, i.e., in $\bar{X}$, for all $\tau \in$ $[0, t]$. Thus, the first switch must occur at $S_{1}$ (see Fig. 20). The inclusion consists of several infinite dimensional sets of linear inequalities, one for each boundary of $X$. For instance, in Fig. 20, it must be true that $C_{j} x(\tau) \geq d_{j}, j=0,1,2$, for all $\tau \in[0, t]$, assuming $C_{j}^{\prime}$ orientations point toward $X$, as in the figure.

In addition, to reduce conservatism the set $S_{0}^{d}$ can exclude those points in $S_{0}$ that cannot be reached by a trajectory of the PLS starting somewhere in $\mathbb{R}^{n} \backslash S_{0}^{d}$, since such points play no role in the stability analysis of the system. Note that the switching surfaces $S_{0}$ and $S_{1}$, together with (1), are part of some larger PLS.

Example 8.1: Fig. 21 shows PLS with both switching surfaces $S_{0}$ and $S_{1}$, and $X$ defined between them. Above the switching surface $S_{0}$, the system is given by $\dot{x}=A_{1} x+B_{1}$. The figure shows the vector fields of systems $\dot{x}=A_{1} x+B_{1}$ and (1) along the switching surface $S_{0}$ (above and below, respectively), and the vector field of (1) along the switching surface $S_{1}$. The points $\bar{x}_{0}, \bar{x}_{1}$, and $\bar{x}_{2}$ are the points where $C_{0}\left(A_{1} \bar{x}_{0}+B_{1}\right)=0, C_{0}\left(A \bar{x}_{1}+B\right)=0$, and $C_{1}\left(A \bar{x}_{2}+B\right)=0$, respectively. Note that $\bar{x}_{1}$ must be to the left of $\bar{x}_{0}$ in order to guarantee existence of solutions.

As seen in Fig. 21, points to the left of $\bar{x}_{1}$ do not belong to the domain of the impact map from $S_{0}$ to $S_{1}$. Also, points in $S_{0}$ between $\bar{x}_{0}$ and $\bar{x}_{1}$ cannot be reached by any trajectory starting somewhere in $\mathbb{R}^{n} \backslash S_{0}$. Thus, only points to the right of $\bar{x}_{0}$ need to be considered for stability analysis purposes. Note that those are exactly the points that can be reached by system $\dot{x}=A_{1} x+B_{1}$. Similarly, only some points to the right of $\bar{x}_{2}$ 


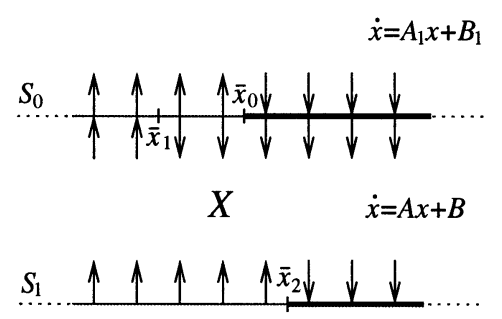

Fig. 21. $S_{0}^{d} \subset S_{0}$ and $S_{1}^{a} \subset S_{1}$ are some sets defined to the right of $\bar{x}_{0}$ and $\bar{x}_{2}$, respectively.

can be reached by (1). Hence, $S_{1}^{a} \subset S_{1}$ is some set defined to the right of $\bar{x}_{2}$.

To exclude points in $S_{0}$ that cannot be reached by a trajectory of the PLS we need a linear equality together with a set of linear inequalities. The equality $C_{0} \Delta_{0}=0$ comes from the fact that $\Delta_{0} \in S_{0}-x_{0}^{*}$. The inequalities are necessary to ensure that every point in $S_{0}^{d}$ can be reached by some trajectory of the PLS, starting in $\mathbb{R}^{n} \backslash S_{0}$. Such points in $S_{0}$ are those where the vector field along $S_{0}$ points inward, for each system $i$ that shares a boundary with $X$ through $S_{0}$ (thicker segments of line in $S_{0}$ in Fig. 22). As in the left of Fig. 22, assume $C_{0}^{\prime}$ orientation points toward $X$ (if this is not the case, just consider $-C_{0}^{\prime}$ and $-d_{0}$ ). The set of points in $S_{0}$ where the vector field of system $i$ is parallel to $S_{0}$ are those where $C_{0} \dot{x}=0$, i.e., $C_{0}\left(A_{i} x+\right.$ $\left.B_{i}\right)=0, x \in S_{0}$. Thus, the set of points in $S_{0}$ that can be reached by system $i$ is some subset of the set of points such that $C_{0}\left(A_{i} x+B_{i}\right)>0, x \in S_{0}$.

\section{B. Less Conservative Conditions}

The aforementioned characterization of $S_{t}$ shows why condition (6) cannot, in general, be written as an equivalent set of LMIs. A straightforward transformation of (6) into a set of LMIs was to use only equality $C_{0} \Delta_{0}=0$. This resulted in a more conservative condition (8). To reduce the conservatism, other inequalities can be incorporate using the S-procedure [20]. The problem is that the S-procedure only results in equivalent, and therefore nonconservative conditions when a quadratic function is subject to a single quadratic constraint. Next, the S-procedure is used to relax (6) to a set of LMIs using equality (3) and other inequalities. We start by incorporating a single inequality.

First, we approximate $S_{t}$ with a larger set. For a given $t \in \mathcal{T}$, let $\tilde{S}_{t} \supset S_{t}$ be the set of points in $S_{0}$ where $C_{1} x(t)=d_{1}$. This can be obtained from (3) yielding

$$
\tilde{S}_{t}=\left\{x_{0}^{*}+\Delta_{0} \in S_{0}: C_{1} e^{A t} \Delta_{0}=d_{1}-C_{1} x_{0}^{*}(t)\right\} .
$$

To see the differences between $S_{t}$ and $\tilde{S}_{t}$, consider again example 3.1. Fig. 23 shows the solution $C_{1} x(t)$ for two different initial conditions in $S_{0}^{d}$.

On the left-hand side of Fig. 23, $t_{\Delta_{0}}=\left\{t_{1}, t_{2}\right\}$. This means $x_{0}^{*}+\Delta_{0}$ belongs to both $S_{t_{1}}$, and $S_{t_{2}}$. On the right-hand side of Fig. 23, $t_{\Delta_{0}}=\left\{t_{1}\right\}$. This figure also shows (dashed) what would have happened to $C_{1} x(t)$ if there was no switch at $t_{1}$. $C_{1} x(t)$ would have intersect $S_{1}$ again at $t=t_{2}$. Although $t_{2}$ is a solution of (3), it is not a valid switching time since $C_{1} x(t)<d_{1}$ for $t_{1}<t<t_{2}$. Thus, $x_{0}^{*}+\Delta_{0}$ belongs to $\tilde{S}_{t_{1}}, S_{t_{1}}$, and $\tilde{S}_{t_{2}}$, but not to $S_{t_{2}}$.

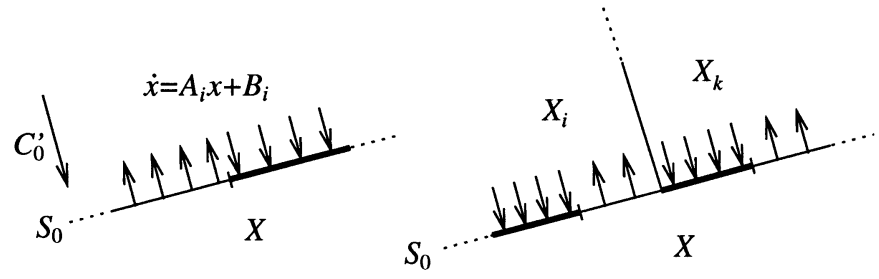

Fig. 22. Points in $S_{0}$ that can be reached by trajectories of the system.
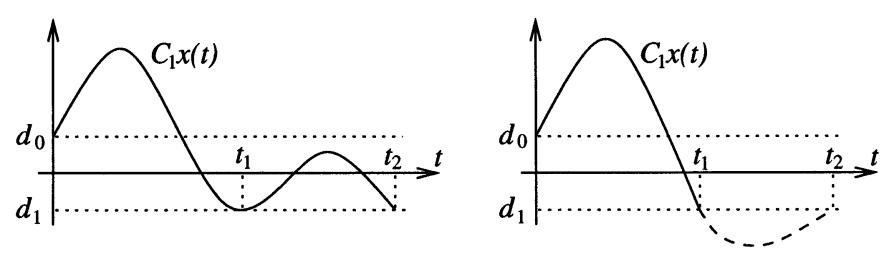

Fig. 23. (Left) $C_{1} x(t) \geq d_{1}$ for $0 \leq t \leq t_{2}$. (Right) $C_{1} x(t)<d_{1}$ for $t_{1}<t<t_{2}$.

Since $S_{t} \subset \tilde{S}_{t}$, condition (6) holds if

$$
R(t)>0 \text { on } \tilde{S}_{t}-x_{0}^{*}
$$

for all switching times $t \in \mathcal{T}$. To obtain a set of LMIs less conservative than (8), consider equality (3) from (20) and any one of the inequalities discussed before, represented here by some $L$ and $m$ such that $L \Delta_{0}>m$. Section VIII-C gives a concrete example with one of these inequalities. A less conservative condition than (22) is then

$$
R(t)>0 \text { on }\left(\tilde{S}_{t} \bigcap\left\{x_{0}^{*}+\Delta_{0} \mid L \Delta_{0}>m\right\}\right)-x_{0}^{*}
$$

for all switching times $t \in \mathcal{T}$ (see Fig. 24).

$\Delta_{0} \in\left(\tilde{S}_{t} \cap\left\{x_{0}^{*}+\Delta_{0} \mid L \Delta_{0}>m\right\}\right)-x_{0}^{*}$ satisfies a conic relation $\Delta_{0}^{\prime} \beta_{t} \Delta_{0}>0$ (Fig. 24), for some matrix $\beta_{t}$ (the construction of this matrix will be addressed in Appendix III). Using the S-procedure, condition (23) is equivalent to

$$
R(t)-\tau_{t} \beta_{t}>0 \text { on } S_{0}-x_{0}^{*}
$$

for some scalar function $\tau_{t}>0$, and for all switching times $t \in \mathcal{T}$. For each $t$, (24) is now an LMI.

By repeatedly using the $S$-procedure, it is possible to continue to improve conditions (24). From the last inclusion in (20), for example, it is necessary that the trajectory $x(\tau)$ stays to the correct side of all switching surfaces that compose the boundary $X$. In particular, it must be true that $C_{1} x(\tau) \geq d_{1}$ for all $\tau \in[0, t]$, i.e.,

$$
C_{1}\left(e^{A \tau} \Delta_{0}+x_{0}^{*}(\tau)\right) \geq d_{1}
$$

for all $\tau \in[0, t]$, which is an infinite-dimensional set of linear inequalities. For example, at $\tau=t / 2$

$$
C_{1} e^{A t / 2} \Delta_{0} \geq d_{1}-C_{1} x_{0}^{*}(t / 2) .
$$

As before, this inequality together with $\tilde{S}_{t}$ satisfies a conic relation $\Delta_{0}^{\prime} \gamma_{t / 2} \Delta_{0}>0$ and (24) is improved to

$$
R(t)-\tau_{t} \beta_{t}-\tau_{1 t} \gamma_{t / 2}>0 \text { on } S_{0}-x_{0}^{*}
$$

for some scalar functions $\tau_{t}, \tau_{1 t}>0$, and for all $t \in \mathcal{T}$.

There is an infinite number of constraints that can be added to condition (25) in order to further reduce the level of conser- 


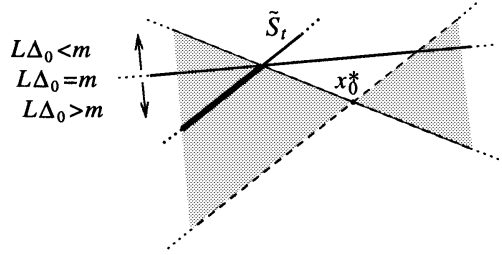

Fig. 24. Region in $S_{0}$, defined by equality (3) and inequality $L \Delta_{0}>m$, satisfies a conic relation.

vatism. On one hand, the more constraints, the less conservative the conditions are and, in turn, better chances of finding SuLF. On the other hand, increasing the number of constraints will eventually make the problem computationally intractable. It is interesting to notice, however, that many important PLS are globally analyzed with just conditions of the form of (8), as seen in [3] and Sections VI and VII.

\section{Application to On-Off}

Next, we explain how the ideas discussed in the previous section can be used to derive less conservative conditions than (14) for on/off systems, similar to those obtained in (21) and (25). Note that all the ideas discussed in this subsection apply analogously to saturation systems.

Define the sets $\tilde{S}_{t_{i}} \supset S_{t_{i}}, i=1,2$, as in (24). In the case of OFS these are $\tilde{S}_{t_{1}}=\left\{x_{0}^{*}+\Delta_{0} \in S: w_{1 t} \Delta_{0}=1\right\}$ and $\tilde{S}_{t_{2}}=\left\{x_{1}^{*}+\Delta_{1} \in S: w_{2 t} \Delta_{1}=1\right\}$.

The domain of impact map 1 is $S_{+}$, which is characterized by all points in $x \in S$ such that $C A x \geq 0$. Similarly, the domain of impact map 2 is a subset of $S_{-}$. The set $S_{-}$is characterized by all points in $x \in S$ such that $C A x \leq 0$. Therefore, conditions (13) hold if

$$
\begin{cases}R_{1}\left(t_{1}\right)>0 & \text { on }\left(\tilde{S}_{t_{1}} \bigcap\{x \mid C A x>0\}\right)-x_{0}^{*} \\ R_{2}\left(t_{2}\right)>0 & \text { on }\left(\tilde{S}_{t_{2}} \bigcap\{x \mid C A x<0\}\right)-x_{1}^{*}\end{cases}
$$

for all switching times $t_{1}, t_{2}$, which are less conservative conditions than (14).

As explained in the previous sections, $\Delta_{i}$ satisfies a conic relation $\Delta_{i}^{\prime} \beta_{t_{i}} \Delta_{i}>0$, for some matrices $\beta_{t_{i}}$ (see Appendix III for details on the construction of such matrices). Using the S-procedure, we obtain equivalent conditions to (26)

$$
\begin{cases}R_{1}\left(t_{1}\right)-\tau_{t_{1}} \beta_{t_{1}}>0 & \text { on } S-x_{0}^{*} \\ R_{2}\left(t_{2}\right)-\tau_{t_{2}} \beta_{t_{2}}>0 & \text { on } S-x_{1}^{*}\end{cases}
$$

for some scalar functions $\tau_{t_{i}}>0$, and for all switching times $t_{i}$. For each $t_{1}, t_{2}$, these conditions are LMIs.

\section{CONCLUSION}

Motivated by the need of new and alternative global analysis tools for certain classes of hybrid systems, this paper develops an entirely new constructive analysis methodology for PLS using impact maps and SuLF. This methodology consists of inferring global properties of PLS solely by studying their behavior at switching surfaces. The main idea is to efficiently construct SuLF to show that impact maps associated with PLS are contracting. The success and power of this new methodology has been demonstrated in globally analyzing equilibrium points and limit cycles of several classes of PLS: relay feedback systems, on/off systems, and saturation systems. A large number of examples of these classes of PLS with a locally stable limit cycle or equilibrium point were successfully globally analyzed. In fact, it is still an open problem whether there exists an example with a globally stable limit cycle or equilibrium point that could not be successfully analyzed with this new methodology.

For the classes of systems considered so far, there were only advantages using SuLF compared with piecewise quadratic Lyapunov functions [2]. SuLF analyzed limit cycles for relay feedback systems, no extra complexity was added (i.e., no need for extra partitions), scaled with the dimension of the system, and could prove global asymptotic stability of PLS that were not exponentially stable. There are, however, limitations to SuLF. It is under investigation how to systematically analyze general PLS, which, at this time, is much simpler in [2]. Also, for equilibrium points, if PLS has a large number of partitions such that the method with [2] does not require extra partitions, then SuLF may be harder to apply due to the large number of impact maps associated with the system.

\section{APPENDIX I}

\section{TECHNICAL DETAILS FOR ON/OFF SYSTEMS}

\section{A. Choice of $x_{0}^{*}$ and $x_{1}^{*}$}

Next, we explain how to choose $x_{0}^{*}$ and $x_{1}^{*}$ such that both $C x_{0}^{*}(t)<d$ and $C x_{1}^{*}(t)<d$ for all $t>0$. We start with $x_{1}^{*}$.

$x_{1}^{*}$ is found as explained in Section VI.A (see the right-hand side of Fig. 8). In this case, $x_{1}^{*} \in S^{*}$ is given by

$$
x_{1}^{*}=\frac{P_{d}^{-1} C^{\prime} d}{C P_{d}^{-1} C^{\prime}}
$$

where $P_{d}>0$ satisfies $P_{d} A+A^{\prime} P_{d}=-I$.

The choice of $x_{0}^{*}$ is more tricky since $A_{1}$ may be unstable. If $A_{1}$ is stable then $x_{0}^{*}$ can be found similarly to $x_{1}^{*}$. Let $P_{u}>0$ satisfy $P_{u} A_{1}+A_{1}^{\prime} P_{u}=-I$. Then

$$
x_{0}^{*}=\left(d+c A_{1}^{-1} B_{1}\right) \frac{P_{u}^{-1} C^{\prime}}{C P_{u}^{-1} C^{\prime}}-A_{1}^{-1} B_{1} .
$$

Assume now that $A_{1}$ is not stable, but has at least one stable eigenvalue. The idea is to find an $x_{0}^{*} \in S$ that belongs to a stable mode of $A_{1}$, so that the trajectory $x(t)$ starting at $x_{0}^{*}$ converges to $-A_{1}^{-1} B_{1}$ and satisfies $C x(t)<d$ for all $t>0$. If $A_{1}$ has real eigenvalues then these must be stable. Let $\lambda$ be a real eigenvalue of $A_{1}$ with associated eigenvector $v$ (assume $C v \neq 0$ ). Then, there exists an $x_{0}^{*} \in S$ that only excites this mode

$$
x_{0}^{*}=-A_{1}^{-1} B_{1}+\frac{d+C A_{1}^{-1} B_{1}}{C v} v .
$$

If $A_{1}$ only has complex poles, pick a stable complex conjugate pair of eigenvalues $\lambda, \underline{\lambda}$ with associated eigenvectors $v, \underline{v}$, where $\underline{x}$ stands for the complex conjugate of $x$. Let, $v_{a}=v+\underline{v}$ and $v_{b}=i(v-\underline{v})$. Then, any initial condition starting in the hyperplane defined by $-A_{1}^{-1} B_{1}+\alpha_{a} v_{a}+\alpha_{b} v_{b}, \alpha_{a}, \alpha_{b} \in \mathbb{R}$ converges to $-A_{1}^{-1} B_{1}$ since it only excites this stable complex conjugate mode. Let $V=\left[v_{a} /\left\|v_{a}\right\| v_{c} /\left\|v_{c}\right\|\right]$ be an orthogonal 
basis in this plane, where $v_{c}=-\left(v_{a}^{\prime} v_{b}\right) v_{a}+v_{b}$. A trajectory in this basis satisfies $\dot{\alpha}=A_{v} \alpha$, where $A_{v}=V^{\prime} A_{1} V \alpha$. We need to find an $\alpha_{0}$ such that $C\left(-A_{1}^{-1} B_{1}+V \alpha_{0}\right)=d$ and $C\left(-A_{1}^{-1} B_{1}+V \alpha(t)\right)<d$ for all $t>0$. This is a similar problem to the one we dealt above when finding $x_{1}^{*}$. In this case, $\alpha_{0}$ is given by

$$
\alpha_{0}=\frac{d+C A_{1}^{-1} B_{1}}{C V P_{v}^{-1} V^{\prime} C^{\prime}} P_{v}^{-1} V^{\prime} C^{\prime}
$$

where $P_{v}>0$ satisfies $P_{v} A_{v}+A_{v}^{\prime} P_{v}=-I$. Finally, $x_{0}^{*}=$ $-A_{1}^{-1} B_{1}+V \alpha_{0}$.

If $A_{1}$ only has complex unstable eigenvalues, then for any choice of $x_{0}^{*}, C x(t)=d$ will have an infinity number of solutions for $t>0$. In this case, $x_{0}^{*}$ must be chosen such that the smallest solution $t>0$ of $C x(t)=d$ is higher than the maximum possible switching time. Note that such $x_{0}^{*}$ may not exist. If that is the case, the linearization of the impact map must be parametrized by another variable at those values of $t \in \mathcal{T}$ where $C x(t)=d$, as explained in Section III.

\section{B. Constraints Imposed When $t_{i}=0$}

Zero switching time corresponds to points in $S$ such that $C A x=0$. At those points, the SuLF must be continuous since this is the only way both $V_{2}\left(\Delta_{1}\right) \leq V_{1}\left(\Delta_{0}\right)$ and $V_{1}\left(\Delta_{2}\right) \leq$ $V_{2}\left(\Delta_{1}\right)$ can be simultaneously satisfied, for all $\Delta_{0}, \Delta_{1}, \Delta_{2}=$ $\Delta_{0}$ such that $x_{0}^{*}+\Delta_{0}=x_{1}^{*}+\Delta_{1}$ and $C A\left(x_{0}^{*}+\Delta_{0}\right)=0$. Thus, at those points, $V_{1}\left(\Delta_{0}\right)=V_{2}\left(\Delta_{1}\right)$, which is equivalent to $R_{0}=R_{1}(0)=R_{2}(0)=0$. This imposes certain restrictions on $P_{1}, P_{2}>0, g_{1}, g_{2}$, and $\alpha$. From Section VI-C, $R_{0}=R_{1}(0)$ is given by

$$
\begin{array}{r}
R_{0}=P_{1}-P_{2}-\left(g_{1}+P_{2} v_{0}-g_{2}\right) w \\
-w^{\prime}\left(g_{1}+P_{2} v_{0}-g_{2}\right)^{\prime}+\bar{\alpha}
\end{array}
$$

where $v_{0}=x_{0}^{*}-x_{1}^{*}, \bar{\alpha}=w^{\prime} w\left(\alpha+2 v_{0}^{\prime} g_{2}-v_{0}^{\prime} P_{2} v_{0}\right)$, and

$$
w \Delta_{0}=\lim _{t \rightarrow 0} w_{1}(t) \Delta_{0}=-\frac{C A}{C\left(A x_{0}^{*}+B d\right)} \Delta_{0} .
$$

Let $l \in w^{\perp}, z=w^{\prime} /\left(w w^{\prime}\right)$, and $P=P_{1}-P_{2}$. Since $R_{0}=0$, then $l^{\prime} R_{0} l=l^{\prime} P l=0$. This means that in the basis $(l, z)$, the matrix $P$ must have the following structure:

$$
P_{F}=\left(\begin{array}{cc}
0 & \Gamma_{1} \\
\Gamma_{1}^{\prime} & \Gamma_{2}
\end{array}\right)
$$

for some $\Gamma_{1} \in \mathbb{R}^{n-1}$ and $\Gamma_{2} \in \mathbb{R}$, where $P=F P_{F} F^{\prime}$, with $F=\left[\begin{array}{ll}l & z\end{array}\right]$. Thus, once $P_{2}>0$ is fixed, $P_{1}>0$ must satisfy $P_{1}=F P_{F} F^{\prime}+P_{2}$. The same way $l^{\prime} R_{0} z=0$, or $l^{\prime}\left(P z-g_{1}-\right.$ $\left.P_{2} v_{0}+g_{2}\right)=0$. Hence, $P z-g_{1}-P_{2} v_{0}+g_{2}=k z$ for some $k \in \mathbb{R}$. For a given $g_{2}, g_{1}$ is then given by

$$
g_{1}=\left(P_{1}-P_{2}\right) z-P_{2} v_{0}+g_{2}-k z .
$$

Finally, it must be true that $z^{\prime} R_{0} z=0$ leading to

$$
\begin{aligned}
\alpha=-z^{\prime}\left(P_{1}-P_{2}\right) z+2 z^{\prime}\left(g_{1}+P_{2} v_{0}-g_{2}\right) & \\
& +v_{0}^{\prime} P_{2} v_{0}-2 v_{0}^{\prime} g_{2} .
\end{aligned}
$$

In conclusion, the constraints imposed when $t_{i}=0$ reduce the free variable in conditions (13) and (14) to $P_{2}, \Gamma_{1}, \Gamma_{2}, g_{2}$, and $k$.

\section{Checking Stability Conditions for $t_{i}>t_{i \max }$}

Just like in RFS [3] and SAT, we would like to obtain bounds on the set of switching times. With the exception of third-order systems, however, finding upper bounds $t_{i \max }$ on switching times is, in general, not an easy task. The idea is to first guarantee conditions (14) are satisfied in some intervals $\left(0, t_{i \max }\right)$ and then check if they are also valid for all $t_{i}>t_{i \max }$. Note that $R_{1}(t) \rightarrow P_{1}$ and $R_{2}(t) \rightarrow P_{2}$ as $t \rightarrow \infty$. Thus, it is guaranteed that (13) and (14) are satisfied at $t=\infty$ since both $P_{1}>0$ and $P_{2}>0$.

For simplicity, we present the case when $d=0$. The other cases follow analogously. Assume conditions (15) are satisfied for all $t_{i} \leq t_{i \max }$. We would like to easily check if they will also be satisfied for all $t_{i}>t_{i \max }$. Consider first the second condition in (15). It is sufficient to show that

$$
\Pi^{\prime} e^{A^{\prime} t_{2}} \Pi Q_{1} \Pi^{\prime} e^{A t_{2}} \Pi<Q_{2}
$$

for all $t>t_{2 \max }$, and where $Q_{i}=\Pi^{\prime} P_{i} \Pi$. Next, we find an upper bound on the left-hand side of the last inequality. Let $A_{z}=\Pi^{\prime} A \Pi$. Since $A$ is a stable matrix, it is possible to find a $Q$ and a $\lambda>0$ such that $Q A_{z}+A_{z}^{\prime} Q<-\lambda Q$. This in turn implies that $z^{\prime}(t) Q z(t)<e^{-\lambda t} z_{0}^{\prime} Q z_{0}$, where $z(t)$ is the solution of $\dot{z}=A_{z} z$ with initial condition $z_{0}$. Using the fact that $e^{A_{z} t}=\Pi^{\prime} e^{A t} \Pi$

$$
z_{0}^{\prime} \Pi^{\prime} e^{A^{\prime} t_{2}} \Pi Q \Pi^{\prime} e^{A t_{2}} \Pi z_{0}<e^{-\lambda t} z_{0}^{\prime} Q z_{0}
$$

or, simply

$$
\Pi^{\prime} e^{A^{\prime} t_{2}} \Pi Q \Pi^{\prime} e^{A t_{2}} \Pi<e^{-\lambda t} Q .
$$

Hence, for some $k$

$$
\begin{aligned}
\Pi^{\prime} e^{A^{\prime} t_{2}} \Pi Q_{1} \Pi^{\prime} e^{A t_{2}} \Pi & <\Pi^{\prime} e^{A^{\prime} t_{2}} \Pi k Q \Pi^{\prime} e^{A t_{2}} \Pi \\
& <k e^{-\lambda t} Q \\
& <k e^{-\lambda t_{2} \max } Q
\end{aligned}
$$

Therefore, we need to guarantee $k e^{-\lambda t_{2 \max }} Q<Q_{2}$, with the largest $\lambda$ and smallest $k$.

If $A_{1}$ is stable, a similar condition can be found analogously for the first condition in (15). However, if $A_{1}$ (or $A$ if $d=0$ ) has unstable complex poles, this approach will not work since $e^{A_{1} t}$ is unbounded when $t \rightarrow \infty$. How to find bounds on switching times for such systems of order higher than 3 is currently under investigation.

\section{APPENDIX II}

\section{TECHNICAL DETAILS FOR SATURATION SYSTEMS: BOUNDS ON SWITCHING TIMES}

This section reduces checking (18) or (19) on some bounded set of switching times $\left(t_{i-}, t_{i+}\right)$, instead of checking them for all possible switching times. In RFS [3], a bounded invariant set where all trajectories eventually enter and stay there was characterized. Bounds on the switching times of trajectories inside that 
bounded invariant set were found. The same ideas can be used for SAT whenever $A$ is Hurwitz since $u= \pm d$ is a bounded input.

First, notice that $t_{1-}=t_{2 a-}=0$ since the associated impact maps are defined on the same switching surface and are allowed to have zero switching time. Analysis of impact maps 1 and $2 a$ at $t_{i}=0$ imposes the same constraints on the parameters of the Lyapunov functions as in OFS. See Appendix I.B for details.

As for impact map $\Delta_{1} \rightarrow \Delta_{2 b}$, zero switching never occurs since there is a "gap" between $S$ and $\underline{S}$, resulting in a nonzero switching time for every trajectory starting in $S_{-d}$. For certain large values of $\left\|\Delta_{1}\right\|$, the switching times can be made arbitrarily small. In the invariant bounded set described above, however, switching times for impact map $2 b$ cannot be made arbitrarily small, and a lower bound $t_{2 b-}$ can be found. Using the same ideas, upper bounds on switching times for all impact maps can be found. Bounds on switching times for the case where $A$ has unstable imaginary eigenvalues can be found as explained in Example 7.3.

Before finding bounds on switching times, we need to characterize a bounded set such that any trajectory will eventually enter and stay there. The following proposition is similar to [3, Prop. 7.1]. Thus, the proof is omitted here.

Proposition 3: Consider the system $\dot{x}=A x+B u, y=F x$, where $A$ is Hurwitz, $u(t)= \pm d$, and $F$ is a row vector. Then

$$
\limsup _{t \rightarrow \infty}|F x(t)| \leq d\left\|F e^{A t} B\right\|_{\mathcal{L}_{1}} .
$$

Remember that, by definition, $\left\|F e^{A t} B\right\|_{\mathcal{L}_{1}}$ is given by

$$
\left\|F e^{A t} B\right\|_{\mathcal{L}_{1}}=\int_{0}^{\infty}\left|F e^{A t} B\right| d t .
$$

As a remark, if $F=C$ and $\left\|F e^{A t} B\right\|_{\mathcal{L}_{1}}<1$, it follows the origin is globally asymptotically stable. When $\limsup _{t \rightarrow \infty}|C x(t)|<d$, eventually all trajectories enter and remain in the set $\{x|| C x \mid<d\}$, where the system is linear and stable. Note that this remark also follows from the well known small-gain theorem.

We first focus our attention on upper bounds of the switching times $t_{i+}$, starting with $t_{1+}$. A trajectory $x(t)$ starting at $x_{0} \in$ $S_{+}$is given by $x(t)=e^{A t}\left(x_{0}+A^{-1} B d\right)-A^{-1} B d$. Thus, the output $y(t)=C x(t)$ is given by

$$
y(t)=C e^{A t}\left(x_{0}+A^{-1} B d\right)-C A^{-1} B d
$$

Since $-C A^{-1} B d<d$ and $A$ Hurwitz, $y(t)$ cannot remain larger than $d$ for all $t>0$. For any initial condition $x_{0} \in$ $S_{+}, C e^{A t}\left(x_{0}+A^{-1} B d\right) \rightarrow 0$ as $t \rightarrow \infty$, which means $y(t)=d$ for some $t$. Thus, a switch must occur in finite time. Since for a sufficiently large enough time $t, x(t)$ enters a bounded invariant set (from the above proposition), an upper bound on this switching time $t_{1}+$ can be obtained. The following proposition is similar to [3, Prop. 7.2].

Proposition 4: Let $t_{1+}>0$ be the smallest solution of

$$
\int_{t_{1+}}^{\infty}\left|C e^{A t} B\right| d t+\left|C e^{A t_{1+}} A^{-1} B\right| \leq\left(C A^{-1} B+1\right)
$$

If $t_{a}$ and $t_{b}$ are sufficiently large consecutive switching times of the first impact map then $\left|t_{a}-t_{b}\right| \leq t_{1+}$.

Next, we find upper bounds on the switching times of impact maps $2 a$ and $2 b$. The idea here is to find the minimum $t_{2} \geq 0$ such that $|y(t)|=\left|C e^{A_{1} t} x_{0}\right| \leq d$, for all $t \geq t_{2}$ and all $x_{0}$ in the bounded invariant set. In this derivation, $t_{2 a+}=t_{2 b+}=t_{2}$.

Proposition 5: Let $t_{2}>0$ be the smallest solution of

$$
\int_{0}^{\infty}\left|C e^{A_{1} t_{2}} e^{A t} B\right| d t \leq 1
$$

If $t_{a}$ and $t_{b}$ are sufficiently large consecutive switching times of impact maps $2 a$ or $2 b$, then $\left|t_{a}-t_{b}\right| \leq t_{2}$, and $t_{2 a+}=t_{2 b+}=t_{2}$.

We now focus on the lower bound on the switching times of impact map $2 b$, i.e, $t_{2 b-}$. Remember that if $x_{0} \in S_{+}$, then $y(0)=d$. Since $d>0$, it must be true that $y(t)>-d$ at least in some interval $(0, \epsilon)$. Basically, the time it takes to go from $S$ to $\underline{S}$ must always be nonzero. The next result shows that when a trajectory enters the bounded invariant set characterized before, $\epsilon$ cannot be made arbitrarily small. Thus, a lower bound on the time it takes between two consecutive switches from $S$ to $\underline{S}$ can be obtained.

Proposition 6: Let $k_{\mathrm{dd}}=\left\|C A_{1}^{2} e^{A t} B\right\|_{\mathcal{L}_{1}}$, and $k_{d l}=\left\|C A_{1} e^{A t} B\right\|_{\mathcal{L}_{1}}$ and define $t_{21}=2 / \sqrt{k_{\mathrm{dd}}}, t_{22}=2 / k_{d l}$. Let $t_{2 b-}=\max \left\{t_{21}, t_{22}\right\}$. If $t_{a}$ and $t_{b}$ are sufficiently large consecutive switching times of impact map $2 b$, then $\left|t_{a}-t_{b}\right| \geq t_{2 b-}$.

The proof is similar to the proof of [3, Prop. 7.3].

\section{APPENDIX III \\ CONSTRUCTION OF CONIC RELATIONS}

We now describe how to construct the cones $\beta_{t}$ introduced in Section VIII-B. For each $t>0$, the cone is defined by two hyperplanes in $S_{0}$ : one is the hyperplane parallel to $\tilde{S}_{t}$ containing $x_{0}^{*}$ and the other is the hyperplane defined by the intersection of $\mathcal{M}=\left\{x_{0}^{*}+\Delta_{0} \in S_{0} \mid L \Delta_{0}=m\right\}$ and $\tilde{S}_{t}$, and containing the point $x_{0}^{*}$ (see Fig. 24). Let $\Pi_{0} l_{t}$ and $\Pi_{0} s_{t}$, respectively, be vectors in $S_{0}$ perpendicular to each hyperplane. Once these vectors are known, the cone can easily be characterized. This is composed of all the vectors $\Delta_{0} \in S_{0}-x_{0}^{*}$ such that $\Delta_{0}^{\prime} \Pi_{0}\left(s_{t} l_{t}^{\prime}+l_{t} s_{t}^{\prime}\right) \Pi_{0}^{\prime} \Delta_{0} \geq 0$. The symmetric matrix $\beta_{t}$ introduced in (24) is just $\beta_{t}=\Pi_{0} \bar{\beta}_{t} \Pi_{0}^{\prime}$ where $\bar{\beta}_{t}=s_{t} l_{t}^{\prime}+l_{t} s_{t}^{\prime}$. Remember that the cone is centered at $x_{0}^{*}$ and note that after $l_{t}$ is chosen, $s_{t}$ must have the right direction in order to guarantee $\left(\tilde{S}_{t} \cap\left\{x_{0}^{*}+\Delta_{0} \mid L \Delta_{0}>m\right\}\right) \subset\left\{x_{0}^{*}+\Delta_{0} \in S_{0} \mid \Delta_{0}^{\prime} \beta_{t} \Delta_{0}>0\right\}$.

We first find $\Pi_{0} l_{t}$, the vector perpendicular to $\tilde{S}_{t}$. Looking back at the definition of $\tilde{S}_{t}, l_{t}$ is given by

$$
l_{t}=\frac{\left(C_{1} e^{A t} \Pi_{0}\right)^{\prime}}{\left\|C_{1} e^{A t} \Pi_{0}\right\|^{2}}\left(d_{1}-C_{1} x_{0}^{*}(t)\right) .
$$

The derivation of $s_{t}$ is not as trivial as $l_{t}$. We actually need to introduce a few extra variables. The first one is $\Pi_{0} l_{0}$, the vector perpendicular to the set $\mathcal{M}$, given by $l_{0}=\left(L \Pi_{0}\right)^{\prime} m /\left\|L \Pi_{0}\right\|^{2}$.

Proposition 7: The hyperplane defined by the intersection of $\mathcal{M}$ and $\tilde{S}_{t}$, and containing the point $x_{0}^{*}$ is perpendicular to the vector

$$
\frac{\Pi_{0} l_{t}}{\left\|l_{t}\right\|}\left\|l_{0}\right\|-\frac{\Pi_{0} l_{0}}{\left\|l_{0}\right\|}\left\|l_{t}\right\|
$$


Proof: $\mathcal{M}$ can be parameterize the following way:

$$
\mathcal{M}=\left\{x_{0}^{*}+\Delta_{0} \in S_{0} \mid \Delta_{0}=\Pi_{0}\left(l_{0}+l_{0}^{\perp} z\right), z \in \mathbb{R}^{n-2}\right\}
$$

and $\tilde{S}_{t}$

$$
\tilde{S}_{t}=\left\{x_{0}^{*}+\Delta_{0} \in S_{0} \mid \Delta_{0}=\Pi_{0}\left(l_{t}+l_{t}^{\perp} w\right), w \in \mathbb{R}^{n-2}\right\} .
$$

The intersection of $\mathcal{M}$ and $\tilde{S}_{t}$ occurs at points in $S_{0}$ such that $l_{0}+l_{0}^{\perp} z=l_{t}+l_{t}^{\perp} w$. Multiplying on the left by $l_{t}^{\prime}$ we have $l_{t}^{\prime} l_{0}+l_{t}^{\prime} l_{0}^{\perp} z=l_{t}^{\prime} l_{t}$ or

$$
l_{t}^{\prime} l_{0}^{\perp} z=\left\|l_{t}\right\|^{2}-l_{t}^{\prime} l_{0} .
$$

We want to show that

$$
\left(\frac{l_{t}}{\left\|l_{t}\right\|}\left\|l_{0}\right\|-\frac{l_{0}}{\left\|l_{0}\right\|}\left\|l_{t}\right\|\right)^{\prime}\left(l_{0}+l_{0}^{\perp} z\right)=0 .
$$

Using (29), we have

$$
\begin{aligned}
& \left(\frac{l_{t}}{\left\|l_{t}\right\|}\left\|l_{0}\right\|-\frac{l_{0}}{\left\|l_{0}\right\|}\left\|l_{t}\right\|\right)^{\prime}\left(l_{0}+l_{0}^{\perp} z\right) \\
& \quad=\frac{l_{t}^{\prime} l_{0}}{\left\|l_{t}\right\|}\left\|l_{0}\right\|+\frac{l_{t}^{\prime} l_{0}^{\perp} z}{\left\|l_{t}\right\|}\left\|l_{0}\right\|-\frac{l_{0}^{\prime} l_{0}}{\left\|l_{0}\right\|}\left\|l_{t}\right\| \\
& =\frac{l_{t}^{\prime} l_{0}}{\left\|l_{t}\right\|}\left\|l_{0}\right\|+\frac{\left\|l_{t}\right\|^{2}-l_{t}^{\prime} l_{0}}{\left\|l_{t}\right\|}\left\|l_{0}\right\|-\left\|l_{0}\right\|\left\|l_{t}\right\|=0 .
\end{aligned}
$$

The characterization of $s_{t}$ is not complete yet. The orientation of $s_{t}$ must be carefully chosen to guarantee that the cone $\mathcal{C}_{t}$ contains $\tilde{S}_{t} \cap\left\{x_{0}^{*}+\Delta_{0} \mid L \Delta_{0}>m\right\}$.

Proposition 8: If

$$
s_{t}=m\left(\frac{l_{0}}{\left\|l_{0}\right\|}\left\|l_{t}\right\|-\frac{l_{t}}{\left\|l_{t}\right\|}\left\|l_{0}\right\|\right)
$$

then the cone $\left\{x_{0}^{*}+\Delta_{0} \in S_{0} \mid \Delta_{0}^{\prime} \beta_{t} \Delta_{0}>0\right\}$ contains $\tilde{S}_{t} \cap$ $\left\{x_{0}^{*}+\Delta_{0} \mid L \Delta_{0}>m\right\}$.

The proof, omitted here, is based on taking a point $\Delta_{0} \in\left(\tilde{S}_{t} \cap\right.$ $\left.\left\{x_{0}^{*}+\Delta_{0} \mid L \Delta_{0}>m\right\}\right)-x^{*}$ and showing that $\Delta_{0}^{\prime} \beta_{t} \Delta_{0}>0$.

\section{REFERENCES}

[1] M. S. Branicky, "Studies in hybrid systems: modeling, analysis, and control," Ph.D. dissertation, Mass. Inst. Technol., Cambridge, MA, 1995.

[2] M. Johansson and A. Rantzer, "Computation of piecewise quadratic Lyapunov functions for hybrid systems," IEEE Trans. Automat. Contr., vol. 43, pp. 555-559, Apr. 1998.

[3] J. M. Gonçalves, A. Megretski, and M. A. Dahleh, "Global stability of relay feedback systems," IEEE Trans. Automat. Contr., vol. 46, pp. 550-562, Apr. 2001.

[4] G. Zames and P. L. Falb, "Stability conditions for systems with monotone and slope-restricted nonlinearities," in SIAM J. Control, vol. 6, 1968, pp. 89-108.

[5] F. D'Amato, A. Megretski, U. Jönsson, and M. Rotea, "Integral quadratic constraints for monotonic and slope-restricted diagonal operators," in Proc. Amer. Control Conf., San Diego, CA, June 1999, pp. 2375-2379.
[6] U. Jönsson and A. Megretski, "The Zames-Falb IQC for systems with integrators," IEEE Trans. Automat. Contr., vol. 45, pp. 560-565, Mar. 2000.

[7] A. Megretski, "New IQC for quasiconcave nonlinearities," presented at the Amer. Control Conf., San Diego, CA, June 1999.

[8] A. Megretski and A. Rantzer, "System analysis via integral quadratic constrains," IEEE Trans. Automat. Contr., vol. 42, pp. 819-830, June 1997.

[9] J. M. Gonçalves, " $\mathcal{L}_{2}$-gain of double integrators with saturation nonlinearity," IEEE Trans. Automat. Contr., vol. 47, pp. 2063-2068, Dec. 2002.

[10] — - "Constructive global analysis of hybrid systems," M.S. thesis, Mass. Inst. Technol., Cambridge, MA, Sept. 2000.

[11] S. Pettersson and B. Lennartson, "An LMI approach for stability analysis of nonlinear systems," presented at the Eur. Control Conf., Brussels, Belgium, July 1997.

[12] A. Hassibi and S. Boyd, "Quadratic stabilization and control of piecewise linear systems," presented at the Amer. Control Conf., Philadelphia, PA, 1998.

[13] H. K. Khalil, Nonlinear Systems, 2nd ed. Upper Saddle River, NJ: Prentice-Hall, 1996.

[14] P. A. Parrilo, "Structured Semidefinite Programs and semialgebraic geometry methods in robustness and optimization," Ph.D. dissertation, California Inst. Technol., Pasadena, CA, 2000.

[15] Web Page. [Online]. Available: http://www.cds.caltech.edu/ jmg/

[16] A. Saberi, Z. Lin, and A. Teel, "Control of linear systems with saturating actuators," IEEE Trans. Automat. Contr., vol. 41, pp. 368-378, Mar. 1996.

[17] H. J. Sussmann, E. D. Sontag, and Y. Yang, "A general result on the stabilization of linear systems using bounded controls," IEEE Trans. Automat. Contr., vol. 39, pp. 2411-2425, Dec. 1994.

[18] A. R. Teel, "A nonlinear small gain theorem for the analysis of control systems with saturation," IEEE Trans. Automat. Contr., vol. 41, pp. 1256-1270, Sept. 1996.

[19] R. Suárez, J. Alvarez, and J. Alvarez, "Linear systems with single saturated input: Stability analysis," presented at the 30th Conf. Decision Control, Brighton, U.K., Dec. 1991.

[20] S. Boyd, L. El Ghaoui, E. Feron, and V. Balakrishnan, Linear Matrix Inequalities in System and Control Theory. Philadelphia, PA: SIAM, 1994.

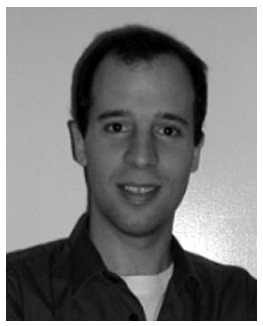

Jorge M. Gonçalves received the Licenciatura (five-year S.B.) degree from the University of Porto, Porto, Portugal, and the M.S. and Ph.D. degrees from the Massachusetts Institute of Technology (MIT), Cambridge, all in electrical engineering and computer science, in 1993, 1995, and 2000, respectively.

Since then, he has held two Postdoctoral positions: first, with MIT for seven months, and since May 2001, with the California Institute of Technology with the Control and Dynamical Systems Division. His research interests include modeling, analysis, and control of complex systems; applications to interdisciplinary fields like biological metabolic networks and circadian rhythms, economic markets, and walking robots; analysis and control of hybrid systems.

Dr. Goncalves was the recipient of the Best Student Paper Award at the Automatic Control Conference, Chicago, IL, June 2000.

Alexandre Megretski was born in 1963. He received the Ph.D. degree from Leningrad University, Leningrad, Russia, in 1988.

He has held research positions at Leningrad University, the Mittag-Leffler Institute and the Royal Institute of Technology, Stockholm, Sweden, and the University of Newcastle, Newcastle, NSW, Australia. From 1993 to 1996, he was an Assistant Professor at Iowa State University, Ames. In 1996, he joined the Massachusetts Institute of Technology, Cambridge, where he is currently an Associate Professor of Electrical Engineering. His research interests include automatic analysis and design of complex dynamical systems, randomized methods of nonconvex optimization, and operator theory. 


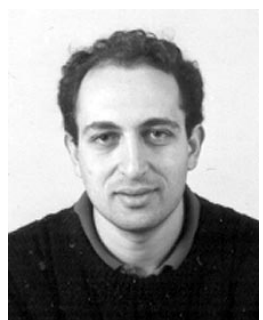

Munther A. Dahleh was born in 1962. He received the B.S. degree from Texas A\&M University, College Station, in 1983, and the Ph.D. degree from Rice University, Houston, TX, in 1987, both in electrical engineering.

Since then, he has been with the Department of Electrical Engineering and Computer Science, the Massachusetts Institute of Technology, Cambridge, where he is now a Full Professor. He was a Visiting Professor at the Department of Electical Engineering, the California Institute of Technology, Pasadena, for spring 1993. He has held consulting positions with several companies in the U.S. and abroad. He is the coauthor (with I. Diaz-Bobillo) of the book Control of Uncertain Systems: A Linear Programming Approach (Upper Saddle River, NJ: Prentice-Hall), and the coauthor (with N. Elia) of the book Computational Methods for Controller Design (Springer-Verlag: NY). His interests include robust control and identification, the development of computational methods for linear and nonlinear controller design, learning from complex data, and applications of feedback control in several disciplines, including material manufacturing and modeling of biological systems.

Dr. Dahleh was the recipient of the Ralph Budd Award in 1987 for the Best Thesis at Rice University, the George Axelby Outstanding Paper Award (paper coauthored with J.B. Pearson in 1987), a National Science Foundation Presidential Young Investigator Award (1991), the Finmeccanica Career Development Chair (1992) and the Donald P. Echman Award from the American Control Council in 1993, and the Graduate Students Council Teaching Award in 1995. He was a Plenary Speaker at the 1994 American Control Conference and at the Mediterranean Conference on Control and Automation in 2003. He was an Associate Editor for the IEEE TRANSACTIONS ON AUTOMATIC CONTROL and for Systems and Control Letters. 